\title{
DPM1 expression as a potential prognostic tumor marker in hepatocellular carcinoma
}

\author{
Ming Li ${ }^{1}$, Shengli Xia ${ }^{\text {Corresp., } 2}$, Ping Shi ${ }^{\text {Corresp. } 1}$ \\ ${ }^{1}$ State Key Laboratory of Bioreactor Engineering, East China University of Science and Technology, Shanghai, China \\ 2 Department of Orthopedics, Shanghai University of Medicine \& Health Sciences Affiliated Zhoupu Hospital, Shanghai, China \\ Corresponding Authors: Shengli Xia, Ping Shi \\ Email address: victorxia@126.com, ship@ecust.edu.cn
}

Background: Altered glycosylation of proteins contributes to tumor progression. Dolichol phosphate mannose synthase (DPMS), an essential mannosyltransferase, plays a central role in post-translational modification of proteins, including N-linked glycoproteins, O-mannosylation, C-mannosylation and glycosylphosphatidylinositol anchors synthesis. Little is known about the function of DPMS in liver cancer.

Methods: The study explored the roles of DPMS in the prognosis of hepatocellular carcinoma using UALCAN, Human Protein Atlas, GEPIA, cBioPortal and Metascape databases. The mRNA expressions of DPM1/2/3 also were detected by quantitative real-time PCR experiments in vitro.

Results: The transcriptional and proteinic expressions of DPM1/2/3 were both over-expressed in patients with hepatocellular carcinoma. Over expressions of DPMS were discovered to be dramatically associated with clinical cancer stages and pathological tumor grades in hepatocellular carcinoma patients. In addition, higher mRNA expressions of DPM1/2/3 were found to be significantly related to shorter overall survival in liver cancer patients. Futhermore, high genetic alteration rate of DPMS (41\%) was also observed in patients with liver cancer, and genetic alteration in DPMS was associated with shorter overall survival in hepatocellular carcinoma patients. We also performed quantitative real-time PCR experiments in human normal hepatocytes and hepatoma cells to verify the expressions of DPM1/2/3 and results showed that the expression of DPM1 was significantly increased in hepatoma cells SMMC-7721 and HepG2.

Conclusions: Taken together, these results suggested that DPM1 could be a potential prognostic biomarker for survivals of hepatocellular carcinoma patients. 
1

2

3

$4 \quad{ }^{1}$ State Key Laboratory of Bioreactor Engineering, East China University of Science and

5 Technology, Shanghai, China

$6 \quad{ }^{2}$ Department of Orthopedics, Shanghai University of Medicine \& Health Sciences Affiliated

7 Zhoupu Hospital, Shanghai, China

8 *To whom correspondence should be addressed. E-mail: ship@ecust.edu.cn(Ping Shi), East China

9 University of Science and Technology, 130 Meilong Road, Shanghai 200237, P. R. China; victorxia@126.com (Shengli Xia), Department of Orthopedics, Shanghai University of Medicine \& Health Sciences Affiliated Zhoupu Hospital, 1500 Zhoupu Zhouyuan Road, Pudong New Area, Shanghai 201318, P. R. China.

13

15

5

16

17

18

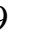

(1)

(2)

(2)

3

(2)

(2)

7

(

\section{(a)}

33

34

35 Abstract 
Background: Altered glycosylation of proteins contributes to tumor progression. Dolichol phosphate mannose synthase (DPMS), an essential mannosyltransferase, plays a central role in post-translational modification of proteins, including N-linked glycoproteins, O-mannosylation, C-mannosylation and glycosylphosphatidylinositol anchors synthesis. Little is known about the function of DPMS in liver cancer.

Methods: The study explored the roles of DPMS in the prognosis of hepatocellular carcinoma using UALCAN, Human Protein Atlas, GEPIA, cBioPortal and Metascape databases. The mRNA expressions of DPM1/2/3 also were detected by quantitative real-time PCR experiments in vitro.

Results: The transcriptional and proteinic expressions of DPM1/2/3 were both over-expressed in patients with hepatocellular carcinoma. Over expressions of DPMS were discovered to be dramatically associated with clinical cancer stages and pathological tumor grades in hepatocellular carcinoma patients. In addition, higher mRNA expressions of DPM1/2/3 were found to be significantly related to shorter overall survival in liver cancer patients. Futhermore, high genetic alteration rate of DPMS (41\%) was also observed in patients with liver cancer, and genetic alteration in DPMS was associated with shorter overall survival in hepatocellular carcinoma patients. We also performed quantitative real-time PCR experiments in human normal hepatocytes and hepatoma cells to verify the expressions of DPM1/2/3 and results showed that the expression of DPM1 was significantly increased in hepatoma cells SMMC-7721 and HepG2. Conclusions: Taken together, these results suggested that DPM1 could be a potential prognostic biomarker for survivals of hepatocellular carcinoma patients.

Keywords: DPMS, liver cancer, biomarker, bioinformatics analysis, prognostic value

\section{Introduction}

Hepatocellular carcinoma (HCC) is one of the most frequently and commonly occurring malignant tumors worldwide. The global incidence and mortality rate of $\mathrm{HCC}$ are ranked 5 th and 3 rd among all types of cancers $(1,2)$. Despite making remarkable advances in new technologies for diagnosis and treatment, the incidence and mortality of HCC still continue to growth because of the poorest prognosis $(3,4)$. Therefore, it is urgently needed to determine reliable predictive biomarkers for early diagnosis and accurate prognosis, and to develop new molecular targeted therapeutic strategies.

The occurrence and development of several cancer types are closely associated with aberrant protein glycosylation $(5,6)$. Studies have suggested that altered glycosylation of proteins has been observed in liver cancer (7). Although mounting evidence has reported the role of glycosylation in tumor progression (8-10), there is limited information on how glycosylation affects the liver cancer development. Recent studies have focused on glycosylation crosstalks with cellular metabolism and related kinases (11-14).

Dolichol phosphate mannose synthase (DPMS), an essential mannosyltransferase, plays a central role in post-translational modification of proteins, including N-linked glycoproteins, Omannosylation, C-mannosylation and glycosylphosphatidylinositol (GPI) of proteins (15). It has three subunits containing DPM1, DPM2 and DPM3 in human. DPM1, a mainly catalytic component of DPMS, is composed of 260 amino acids without any transmembrane domain 
region $(16,17)$. DPM2 and DPM3 are regulatory subunits that help DPM1 localize on the endoplasmic reticulum membrane and enable it to exert catalytic activity (18). The most reported about DPMS gene is that its absence activity is associated with congenital diseases of glycosylation (CDG) and a defect in DPM1 has been indentified to cause CDG-Ie $(19,20)$. In addition to this, studies have reported that abnormal expression or altered enzymatic activity of DPMS was related to cell proliferation and angiogenesis. Increased DPMS activity in bovine capillary endothelial cells correlated with rised cellular proliferation (21). Moreover, previous studies also reported that overexpressing DPMS in capillary endothelial cells significantly enhanced angiogenesis and strengthened wound healing (22). DPMS activity however, was lacking and subsquently led to cell cycle arrest and induction of apoptosis in tunicamycin-treated capillary endothelial cells (23). Reduced gene expression of DPMS also decreased the cellular angiogenic potential (24). These research results indicate that the genes encoding DPMS and its protein activity may be positively related to tumor progression. However, the specific role of DPMS remains unclear in the development and progression of liver cancer. In this present work, we solved this problem by analyzing the expressions and genetic alterations of three subunits of DPMS and their association with clinical parameters in HCC patients. Furthermore, we also analyzed the predicted functions and pathways of DPMS as well as their similar genes.

\section{Materials and methods}

\section{Datasets}

Datasets used for correlation analysis between DPM1/2/3 and chronic liver disease (CLD) were obtained from GEO database (http://www.ncbi.nlm.nih.gov/geo/) after searching for keywords related to CLD. We selected three separate gene expression profiles (GSE114783, GSE128726 and GSE89632) for our study and the detailed information of the datasets was shown in Table 1. The data used for ROC curve plotted were collected from TCGA LIHC datasets. The figures about the relationship between DPM1/2/3 expression and CLD were drawn using R package, ggplot2 v3.3.2. The significance of DPM1/2/3 expressions between normal and CLD samples were analyzed via unpaired Student's $t$-test. The ROC curves were created by R package, pROC v1.16.2.

\section{UALCAN}

UALCAN (http://ualcan.path.uab.edu) is a comprehensive, user-friendly, and interactive web resource and provides data online analysis and mining based on cancer OMICS data (TCGA and MET500). It is designed to analyze relative transcriptional expression of potential genes of interest between tumor and normal samples and association of the transcriptional expression with relative clinicopathologic parameters. In addition, it is also used to evaluate epigenetic regulation of gene expression and pan-cancer gene expression (25). In our study, UALCAN was used to analyze the mRNA expressions of three subunits of DPMS in HCC samples and their relationship with clinicopathologic parameters. Difference of transcriptional expression or pathological stage analysis was compared by Student's $t$-test and $\mathrm{p}<0.05$ was considered as statically significant.

\section{Human Protein Atlas}


116 The Human Protein Atlas (https://www.proteinatlas.org) is a website that provides human

117 proteins data in cells, tissues and organs, including immunohistochemistry-based expression data

118 for near 20 common kinds of cancers (26). The database can be conveniently used to compare

119 the protein differential expressions of interest genes in tumors and normal tissues. In this study,

120 direct comparison of protein expression of three subunits of DPMS between human normal and

121 HCC tissues was performed by immunohistochemistry image.

\section{GEPIA}

123

124

125

126

127

128

129

130

131

132

133

134

135

136

137

138

139

140

141

142

143

144

145

146

147

148

149

150

151

152

153

154

155

156

Gene Expression Profiling Interactive Analysis (GEPIA) is a database developed and built by the team of professor Zhang of Peking University based on the data of the UCSC Xena project. It is an interactive web server that can dynamically analyze and visualize TCGA (The Cancer Genome Atlas) gene expression profile data. It can provide customizable and powerful functions, including differential expression analysis between tumor and normal samples, profiling plotting, survival analysis, similar gene detection, and so on (27). In the current study, we operated correlative prognostic analysis and similar gene detection of DPM1, DPM2 and DPM3, respectively. $\mathrm{p}<0.05$ was considered as statically significant. The significance of expression analysis was completed using Student's $t$-test. Kaplan-Meier curve was used to accomplish prognostic analysis.

\section{cBioPortal}

cBioPortal (www.cbioportal.org), an online open-access website resource, can display multidimensional cancer genomics data in a visual form. It can also help researchers explore the genetic changes between samples, genes and pathways, and combine them with clinical results (28). In this experiment, we studied the genomic profiles of DPMS three subunits, which included putative copy-number alterations (CNAs) from genomic identification of significant targets in cancer (GISTIC) and mRNA Expression z-Scores (RNASeq V2 RSEM) were gained with a z-score threshold \pm 1.8 . Genetic alterations in DPMS and their association with overall survival (OS) and disease free survival (DFS) of HCC patients were exhibited as Kaplan-Meier plots and log-rank test was implemented to confirm the significance of the difference between the survival curves, and when a $p$ value $<0.05$, the difference was statically significant.

\section{Metascape}

Metascape (http://metascape.org), a free and credible gene-list analysis device, can be used for gene annotation analysis and function analysis. It is a mechanized meta-analysis device that can realize habitual and different pathways in a set of orthogonal target-discovery studies (29). In this work, Metascape was used to implement function and pathway enrichment analysis of DPMS members and their similar genes that acquired using GEPIA. Statistically significant difference was $p<0.05$ and minimum enrichment number was 3. Databases containing OmniPath and BioGrid were used for protein-protein interactions enriched analysis. Futhermore, Molecular Complex Detection (MCODE) was supposed to recognize closely related protein components.

\section{Cell Culture}

The human hepatoma cells SMMC-7721, HepG2 and immortal hepatic cell QSG-7701 involved in the experiment were gained from Institute of Cell Biology (Shanghai, China). All cell lines were 
157 cultured in RPMI-1640 or DMEM medium (Gibco/Invitrogen, Camarillo, CA, UNITED 158 STATES) supplied with $10 \%$ fetal bovine serum (PAN-Biotech, Aidenbach, Germany), and then 159 all cells were incubated at $37^{\circ} \mathrm{C}$ in a $5 \% \mathrm{CO} 2$ environment.

160 RT-qPCR

161 TRIeasy ${ }^{\mathrm{TM}}$ Total RNA Extraction Reagent (Yeasen, Shanghai, China) was used for total RNA

162 extraction, and then the total RNA was reverse transcribed to cDNA with the Hifairß II 1st Strand

163 cDNA Synthesis Kit (Yeasen, Shanghai, China) according to the product instruction. Hieff 164 UNICON® Power qPCR SYBR Green Master Mix (Yeasen, Shanghai, China) was used to 165 conduct RT-qPCR experiment on a Bio-Rad CFX96 System (Bio-Rad, Hercules, CA, USA). The 166 reaction conditions were as follows: pre-denaturation at $95^{\circ} \mathrm{C}$ for $30 \mathrm{~s}$, followed by 40 cycles of 167 amplification at $95^{\circ} \mathrm{C}$ for $10 \mathrm{~s}$ and $60^{\circ} \mathrm{C}$ for $30 \mathrm{~s}$. Relative mRNA expression levels of DPM1/2/3 168 were measured based on the $2^{-\Delta \Delta \mathrm{Ct}}$ method with $18 \mathrm{~S}$ used for normalization. The significance of 169 expression analysis was completed using Student's $t$-test. Table 2 showed the primers we used in 170 this study.

171 Results

172 1. Transcriptional levels of DPMS in liver cancer

In order to explore the gene expressions of three subunits of DPMS in different types of cancer, mRNA expressions of DPM1, DPM2 and DPM3 were analyzed by UALCAN. As was shown in Figure 1, we observed that DPM1, DPM2 and DPM3 had higher mRNA expressions for most kinds of tumor samples compared to normal samples, respectively. For example, mRNA expression levels of DPM1 and DPM2 were very highly expressed in colon adenocarcinoma (COAD) (DPM1, p=1.62E-12; DPM2, p<1E-12), head and neck squamous cell carcinoma (HNSC) (DPM1, p <1E-12; DPM2, p=1.62E-12 ), esophageal carcinoma (ESCA) (DPM1, $\mathrm{p}=1.22 \mathrm{E}-07$; DPM2, $\mathrm{p}=2.30 \mathrm{E}-02$ ), liver hepatocellular carcinoma (LIHC) (DPM1, p=1.62E-12; DPM2, p<1E-12 ), rectum adenocarcinoma (READ) (DPM1, p=4.07E-09; DPM2, p=1.62E-12 ) and so on (Figure 1A,B). Similarly, DPM3 gene was particularly highly expressed in breast invasive carcinoma (BRCA) $(p=1.62 \mathrm{E}-12)$, ESCA ( $p=8.22 \mathrm{E}-10)$, LIHC $(p=1.11 \mathrm{E}-16)$ and glioblastoma multiforme (GBM) $(p=1.53 \mathrm{E}-05)$ (Figure 1C). Thus, our results showed that transcriptional expressions of DPMS were significantly over-expressed in many different types of cancer. In particular, all three subunits of DPMS were expressed highly in LIHC and ESCA. Next, we examined the specific mRNA expressions of DPM1, DPM2 and DPM3 in liver tumor using UALCAN database. As was shown in Figure 2A,B and C, mRNA expressions of three genes were all found significantly up-regulated in HCC tissues compared to normal samples (all $\mathrm{p}<0.001)$. We next performed the protein expression levels of DPMS in HCC using Human Protein Atlas database. Results indicated that medium and low protein expressions of DPM1 and DPM3 were expressed in normal liver tissues, while high protein expressions of them were showed in HCC tissues (Figure 2D,F). In addition, DPM2 protein were not detected in normal liver tissues, whereas medium expression of DPM2 were observed in HCC tissues (Figure 2E). In general, the results indicated that transcriptional and proteinic expressions of DPMS were both 
196

197

198

199

200

201

202

203

204

205

206

207

208

209

210

211

212

213

214

215

216

217

218

219

220

221

222

223

224

225

226

227

228

229

230

231

232

233

234

235

236

over-expressed in patients with HCC.

\section{Relationship between the mRNA levels of DPMS and the clinicopathological parameters in liver cancer patients}

Because we observed mRNA and protein levels of DPMS were over-expressed in HCC patients, we subsequently investigated the connection between mRNA expressions of DPMS members with clinicopathological features of HCC patients with UALCAN, containing tumor grades and patients' individual cancer stages. As presented in Figure 3, mRNA expressions of DPMS members were significantly associated with tumor grades, and the mRNA expressions of DPMS headed to be higher with tumor grade elevated. The maximum mRNA expressions of DPM1/2 were showed in tumor grade 4 (Figure 3A,B), whereas the supreme mRNA expression of DPM3 was found in tumor grade 3 (Figure 3C). The reason why mRNA expression of DPM3 in grade 3 seemed to be higher than that in grade 4 may be attributed to the small sample size (only 12 HCC patients at grade 4). Similarly, the mRNA expressions of DPMS were noticeably related to the cancer stage of patients so, the patients with more advanced cancer, the higher in mRNA expressions of DPMS. The highest mRNA expressions of DPM1/2 were observed in tumor stage 3 (Figure 3D,E), while the maximum DPM3 mRNA expression was noticed in stage 4 (Figure 3F). Briefly, the results above indicated that mRNA expressions of DPMS were obviously associated with pathological parameters in HCC patients. Moreover, HCC usually developed from CLD caused by hepatitis B virus (HBV) and hepatitis $\mathrm{C}$ virus (HCV) infection, fatty liver and so on. The relationships between the expressions of DPM1/2/3 and CLD including HBV-related liver cirrhosis, HCV-related liver cirrhosis and non-alcoholic steatohepatitis were also analyzed. Three suitable datasets (GSE114783, GSE128726 and GSE89632) were chosen for verifying the expression of DPM1/2/3 in CLD. We found that the expressions of DPM1/2/3 in HBV and HCV-related liver cirrhosis and non-alcoholic steatohepatitis samples were more or less higher than normal samples (Figure 4). Therefore, the expressions of DPM1/2/3 were also related to disease development of HCC.

\section{Prognostic value of mRNA expression of DPMS in liver cancer patients}

To assess the value of differentially expressed DPMS in the progression of HCC, we used GEPIA to evaluate the relationship between differentially expressed DPMS and clinical outcome. OS curves were presented in Figure 5. We detected that liver cancer patients with low transcriptional levels of DPM1 $(p=0.007)$, DPM2 $(p=0.0032)$ and DPM3 $(p=0.029)$, were significantly connected with longer OS (Figure 5A,B and C). The worth of differentially expressed DPMS in the DFS of HCC patients was also estimated. Noteworthy, the longer DFS indicated to the HCC patients with lower DPM2 transcriptional levels $(p=0.049)$ (Figure 5E). The receiver operating characteristic (ROC) curves were used to detect the prediction accuracy of DPM1/2/3 in distinguishing the HCC from the normal samples compared with existed liver tumor markers containing alpha-fetoprotein (AFP), glypican-3 (GPC-3) and transforming growth factor- $\beta 1$ (TGF $\beta 1$ ). Our results indicated that DPM1/2/3 had a better performance than AFP and TGF $\beta 1$ for the diagnosis of HCC (Figure 6). Area under the curve (AUC) of the DPM1/2/3 were $0.709,0.860$ and 0.746 , respectively (Figure 6A,B and C). AUC of the existed tumor markers including AFP, GPC-3 and TGF $\beta 1$ were $0.679,0.879$ and 0.577 , respectively (Figure 6D,E and 
237 F). Taken together, DPM1/2/3 may be also potential biomarkers for diagnosis or screening of

238 HCC besides AFP, GPC-3 and TGF $\beta 1$.

239 4. DPMS genetic alteration and similar gene network in patients with $\mathrm{HCC}$

Next, we implemented a universal analysis of the molecular characteristics of differentially expressed DPMS. Genetic variations of differentially expressed DPMS in HCC was analyzed utilizing cBioPortal. A total of 366 samples from TCGA pan cancer database were studied, and altered gene set or pathway was detected in 151queried samples (alteration rate was $41 \%$ ). The alteration rates of DPM1, DPM2, and DPM3 were 19\%,6\% and 24\%, respectively ((Figure 7A,B). The most prevalent change in these samples was enhanced mRNA expression. The Kaplan-Meier plotter results and log-rank test presented a considerable difference in OS $(p=0.0264)$, but no remarkable difference in DFS $(p=0.0841)$ between the samples with changes in one of the target genes and those without variations in any target genes (Figures 7C,D).

\section{Functional enrichment analysis of DPMS in patients with HCC}

Top 50 genes similar to DPM1, DPM2 and DPM3 respectively (a total of 150 genes) were searched by GEPIA (Supplementary Table 1). Next, the functions of DPMS and their similar genes were predicted by analyzing GO and KEGG in Metascape. The top 20 GO enrichment items were classified into three functional groups: biological process group, molecular function group, and cellular component group (Figures 8A,B and Table 3). The DPMS members and their similar genes were mainly enriched in biological processes such as ncRNA processing, DNA repair, viral gene expression, deoxyribonucleoside triphosphate metabolic process and so on. The molecular functions regulated by DPMS and their similar genes were snRNP binding, ubiquitin binding, nucleotidyltransferase activity and ubiquitin-like protein transferase activity. The cellular components affected by DPMS and their similar genes were involved in transferase complex, methyltransferase complex, chromosomal region and nucleolar part.

The 6 most significant KEGG pathways for the DPMS and their similar genes were displayed in Figures 8C,D and Table 4. These pathways comprised pyrimidine metabolism, RNA transport, ubiquitin mediated proteolysis, mTOR signaling pathway and so on. Moreover, for more comprehending the relationship between DPMS and HCC, we performed enrichment analysis of protein-protein interaction with Metascape. Figures $8 \mathrm{E}$ and $\mathrm{F}$ exhibited the protein interaction correlation and important MCODE components. The top 3 essential MCODE components were achieved from the protein-protein interaction network. After function and pathway enrichment analysis for each MCODE constituents respectively, the results demonstrated that biological functions regulated by DPMS and their similar genes were mainly related to mRNA and RNA splicing, protein export form nucleus and nucleocytoplasmic transport.

\section{The mRNA expression levels of DPM1/2/3 in vitro}

We evaluated DPM1, DPM2 and DPM3 expression levels in a panel of three cell lines: two hepatoma cells (HepG2 and SMMC-7721) and one normal liver cell line (QSG-7701). The mRNA expression measured by RT-qPCR revealed that DPM1 transcription levels in cancerous cell lines were higher than that in normal liver cells (Figure 9A) and the result was consistent with our prediction. Moreover, the expression of DPM2 and DPM3 in SMMC-7721 cell was 
278 significantly increased, while those expression did not change significantly in HepG2 cell

279 (Figure 9B,C). This discrepancy may be due to a number of differences between cell types and

280 more cell and tissue samples are needed to validate the results. Therefore, DPM1 could be the

281 most potential prognostic biomarker for survivals of HCC patients.

\section{Discussion}

Abnormal glycosylation has been found in human cancer cells decades ago, and more and more researchers have discovered that protein glycosylation contributed to tumor metastasis, angiogenesis and progression (30,31). Being an essential component of glycosyltransferase complex, DPMS protein is involved in multiple protein glycosylation process, including Nglycosylation, O-glycosylation, C-mannosylation and GPI anchors synthesis (15). Many studies have reported that overexpressed DPMS promoted cell proliferation and angiogenesis (22), and silencing DPMS with shRNA significantly reduced cell growth (24). Moreover, increased DPMS activity also accelerated cellular growth $(21,23)$. In view of the above results, we speculated that DPMS may be related to tumorigenesis and progression. To confirm this hypothesis, we predicted the expression of DPMS in cancer through bioinformatics methods, especially in liver cancer. In addition, genetic alteration and prognostic values of three subunits of DPMS in HCC were also analyzed.

Results from our study showed that the transcriptional levels of DPMS were highly expressed in different types of cancer. Moreover, over-expressions of mRNA and protein were both found in three subunits of DPMS, and mRNA expressions of DPMS were significantly associated with patients' individual cancer stages and tumor grades in HCC patients. Besides, higher mRNA expressions of DPM1/2/3 were significantly associated with shorter OS in liver cancers patients. Meanwhile, higher mRNA expression of DPM2 was significantly associated with shorter DFS in liver cancers samples. These data demonstrated that differentially expressed DPMS may play a significant role in HCC. Since three subunits of DPMS were significantly differentially expressed in HCC and closely related to liver tumor prognosis, we next explored their molecular characteristics in HCC. High alteration rate (41\%) of DPMS was observed in HCC patients and the genetic alteration in DPMS was associated with shorter OS in HCC patients. Tumorigenesis and development of $\mathrm{HCC}$ is sophisticated and various, and genetic alteration exerts an important function among this process (32). Among the genetic alteration elevated mRNA expression and gene amplification were the most common changes. Gene amplification, or genomic DNA copy number aberration, is frequently observed in some solid tumors and has been thought to contribute to tumor evolution (33-35). Therefore, the high alteration of gene amplification in DPMS may be related to liver cancer progression. However, the specific function of gene amplification of DPMS in liver cancer need to be further studied. Finally, functions and pathways of DPM1/2/3 and their total 150 similar genes in HCC patients were analyzed. Biological processes such as ncRNA processing and DNA repair, cellular components such as transferase complex, molecular functions snRNP binding and ubiquitin binding, signal pathways such as RNA transport were remarkably regulated by DPMS and their similar genes in HCC. Our findings that DPMS was highly expressed in tumor cells are consistent with the conclusion that overexpression of DPMS in capillary endothelial cells 
319 promoted cell proliferation (22). In addition, a paper noted that upregulation of DPMS activity 320 may involve in angiogenesis for breast and other solid tumor proliferation and metastasis and 321 identified DPMS as a potential "angiogenic switch" (21). Another report related to prostate 322 tumor invasion pointed out DPM3 was a invasion suppressor using microarray expression with our conclusion that DPM3 was over-expressed in liver cancer cells, and the relationship between DPM3 and the invasion ability in liver cancer cells is worth further study. In addition to the above, the abnormal expressions of DPMS have been reported to be associated with human health, such as aging (37), Thy-1 lymphoma (38) and CDG $(19,39)$. These findings may help us to deepen our understanding for the role of DPMS in tumorigenesis and specific action mechanism among cancers.

It is known that HCC generally occurs in patients withCLD as a result of HBV and HCV infections, nonalcoholic fatty liver disease and alcohol-use disorder (40). The occurrence of CLD caused by above factors is related to the glycosylation changes of key proteins (41-45). For example, hepatocytes in transgenic mice that specifically expressed Nacetylglucosaminyltransferase III (GnT-III) had a swollen oval-like morphology and many lipid droplets $(41,42)$. GnT-III was also likely to play essential roles in the change of glycosylation in viral infected people with liver diseases. DPMS is upstream of GnT-III and whether DPMS participates in the regulation process of this enzyme is worth further studying. In addition, ethanol oxidation products such as acetaldehyde interfered with the $\mathrm{N}$-glycan biosynthesis and/or transfer by binding the involved enzymes in patients with liver disease. Modified glycosylation influenced proteins and receptors binding of the sinusoidal and cell surfaces of the liver in diverse CLD. Main membrane receptors glycosylation orchestrated their function in controlling tumor cell adhesion, motility and invasiveness (43). Furthermore, modification in glycosylated receptor assignment and concentration led to glycoproteins accumulation, which were associated with the tumor size in HCC patients $(44,45)$. Hence, the etiology of liver cancer due to chronic liver disease is perhaps attributed to the major membrane receptors and DPMS as an essential mannosyltransferase may be involved in glycosylation of major membrane receptors in liver cancer.

Meanwhile, alterations in glycosylation are a common feature of cancer cells, and the complexity in protein glycosylation improves cell molecules functional diversity (46). Many glycosyltransferases such as N-acetylglucosaminyltransferase V (GnT-V), N-acetylglucosaminyltransferase III (GnT-III) and $\alpha 1-6$ fucosyltransferase (FUT8) have been considered to be related to the development of HCC. Genomic analysis of HCC patients inspired that overexpressed of FUT8 gene, the cause of core fucosylation, indicated that these glycan changes promoted hepatocarcinogenis, letting them potential tumor biomarkers and therapeutic targets (47). Studies have shown that expression changes of fucosyltransferase 1 and $\beta-1,3-$ galactosyltransferase 5 led to the occurrence of HCC (48). High expression of these enzymes in liver cancer patients was closely linked to shorter survival times of HCC patients (49). DPMS is upstream of these enzymes and the expression of DPMS is closely related to the expression of these enzymes. Therefore, DPMS may influence prognosis of $\mathrm{HCC}$ via affecting these related 
enzymes or similar mechanisms with these enzymes.

So far AFP, des- $\gamma$-carboxy-prothrombin (DCP), GPC3 and TGF $\beta 1$ are the major alreadyexisted cancer biomarkers for HCC (50). These biomarkers could be used for early detection of HCC and as markers of recurrence in the follow-up of HCC patients. AFP is more sensitive to the diagnosis of HCC, but its specificity is lower than that of DCP $(51,52)$. Soluble GPC3 is more sensitive than AFP in monitoring highly or moderately differentiated HCC. Simultaneous detection of two or more markers increases the overall sensitivity from $50 \%$ to $72 \%(53)$. However, about $30 \%$ of HCC patients are still negative for these traditional tumor markers. In our study, DPM1 could be a potential prognostic biomarker for survivals of HCC patients. Therefore, it is possible to use DPM1 as an effective supplemental biomarker of liver cancer. The combined application of DPM1 and other already-existed biomarkers would greatly improve the early diagnosis and accurate prognosis of liver cancers. Our study also has some limitations. First, despite mRNA expressions of DPM1/2/3 were related to the prognosis of HCC, all the data performed in our research were obtained from the online website, further studies containing larger sample sizes are needed to confirm our results and to explore the clinical application of the DPMS in HCC treatment. Second, we did not assess the potential diagnostic and therapeutic roles of DPMS in HCC, so future studies are required to explore whether DPMS could be used as diagnostic markers or as therapeutic targets. Finally, we did not explore the potential mechanisms of DPMS in HCC. Future studies are worth to investigate the detailed mechanism between DPMS expression and HCC.

\section{Conclusion}

In this paper, we studied the expressions of DPM1/2/3 in tumor cells and its relationship with tumorigenesis for the first time. Our results showed that over-expressions of DPM1/2/3 were significantly associated with clinical cancer stages and pathological tumor grades in HCC patients. Besides, higher mRNA expressions of DPM1/2/3 were found to be significantly connected with OS in HCC patients. Moreover, high genetic alteration rate of DPM1/2/3 (41\%) was also observed, and genetic alteration in DPM1/2/3 was associated with shorter OS in HCC patients, which provide a better understanding of molecular targets for improved liver cancer therapeutic strategies in the future. DPM1 was the most potential prognostic biomarker for liver cancer via cell experiment verified. To sum up, these results indicated that DPM1 could be a prognostic biomarker for survivals of HCC patients.

\section{Acknowledgments}

This work was sponsored by grants from the National Natural Science Foundation of China (31671309), and the Training Planned Fund of Academic Leaders, Shanghai Pudong New Area Health System (PWR 12018-09).

\section{Competing Interests}

The authors declare no competing interests.

\section{Author Contributions}

Designing and Writing, ML; Visualization, SX and PS; Supervision, ML, SX and PS; Funding Acquisition, SX and PS.

\section{Data Availability}


401 The author confirms that data from the public database for this study have explained the source of

402 the data in detail in the manuscript and provided a link address.

403

404

405

406

407

408

409

410

411

412

413

414

415

416

417

418

419

420

421

422

423

424

425

426

427

428

429

430

431

432

433

434

435

436

437

438

439

440

441

\section{References}

1. Jemal A, Bray F, Center MM, Ferlay J, Ward E, Forman D. Global cancer statistics. CA Cancer J Clin. 2011;61(2):69-90. https://doi.org/10.3322/caac.20107

2. Siegel R, Ma J, Zou Z, Jemal A. Cancer statistics. CA Cancer J Clin. 2014;64(1):9-29. https://doi.org/10.3322/caac.21208

3. Llovet JM, Burroughs A, Bruix J. Hepatocellular carcinoma. Lancet. 2003;362(9399):19071917. https://doi.org/10.1016/S0140-6736(03)14964-1

4. Maluccio M, Covey A. Recent progress in understanding, diagnosing, and treating hepatocellular carcinoma. CA Cancer J Clin. 2012;62(6):394-399. https://doi.org/10.3322/caac.21161

5. Pinho SS, Reis CA. Glycosylation in cancer: mechanisms and clinical implications. Nat Rev Cancer. 2015;15(9):540-555. https://doi.org/10.1038/nrc3982

6. Stowell SR, Ju T, Cummings RD. Protein glycosylation in cancer. Annu Rev Pathol. 
2015;10:473-510. https://doi.org/10.1146/annurev-pathol-012414-040438

7. Mehta A, Herrera H, Block T. Glycosylation and liver cancer. Adv Cancer Res. 2015;126:257279. https://doi.org/10.1016/bs.acr.2014.11.005

8. Fuster MM, Esko JD. The sweet and sour of cancer: glycans as novel therapeutic targets. Nat Rev Cancer. 2005;5(7):526-542. https://doi.org/10.1038/nrc1649

9. Hakomori S. Glycosylation defining cancer malignancy: new wine in an old bottle. Proc Natl Acad Sci U S A. 2002;99(16):10231-10233. https://doi.org/10.1073/pnas.172380699

10. Reis CA, Osorio H, Silva L, Gomes C, David L. Alterations in glycosylation as biomarkers for cancer detection. J Clin Pathol. 2010;63(4):322-329. https://doi.org/10.1136/jcp.2009.071035

11. Butt AM, Feng D, Idrees M, Tong Y, Lu J. Computational identification and modeling of crosstalk between phosphorylation, $\mathrm{O}-\beta$-glycosylation and methylation of FoxO3 and implications for cancer therapeutics. Int J Mol Sci. 2012;13(3):2918-2938. https://doi.org/10.3390/ijms13032918

12. Wang X, Li D, Wu G, Bazer FW. Functional roles of fructose: crosstalk between O-linked glycosylation and phosphorylation of Akt-TSC2-MTOR cell signaling cascade in ovine trophectoderm cells. Biol Reprod. 2016;95(5):102. https://doi.org/10.1095/biolreprod.116.142281 13. Itkonen HM, Mills IG. N-linked glycosylation supports cross-talk between receptor tyrosine kinases and androgen receptor. PLoS One. 2013;8(5):e65016. https://doi.org/10.1371/journal.pone.0065016

14. Nguyen AT, Chia J, Ros M, Hui KM, Saltel F, Bard F. Organelle specific O -Glycosylation drives MMP14 activation, tumor growth, and metastasis, Cancer Cell. 2017;32(5):639-653 e636. https://doi.org/10.1016/j.ccell.2017.10.001

15. Maeda Y, Kinoshita T. Dolichol-phosphate mannose synthase: Structure, function and regulation. Biochim Biophys Acta. 2008;1780(6):861-868. https://doi.org/10.1016/j.bbagen.2008.03.005

16. Tomita S, Inoue N, Maeda Y, Ohishi K, Takeda J, Kinoshita T. A Homologue of Saccharomyces cerevisiae Dpm1p Is Not Sufficient for Synthesis of Dolichol-Phosphate-Mannose in Mammalian Cells. J Biol Chem. 1998;273(15):9249-9254. https://doi.org/10.1074/jbc.273.15.9249

17. Colussi PA, Taron CH, Mack JC. Human and Saccharomyces cerevisiae dolichol phosphate mannose synthases represent two classes of the enzyme, but both function in Schizosaccharomyces pombe. Proc Natl Acad Sci U S A. 1997;94(15):7873-7878. https://doi.org/10.1073/pnas.94.15.7873

18. Maeda Y, Tanaka S, Hino J, Kangawa K, Kinoshita T. Human dolichol-phosphate- mannose synthase consists of three subunits, DPM1, DPM2 and DPM3. EMBO J. 2000;19(11):2475-2482. https://doi.org/10.1093/emboj/19.11.2475

19. Kim S, Westphal V, Srikrishna G, Mehta DP, Peterson S, Filiano J, Karnes PS, Patterson MC, Freeze HH. Dolichol phosphate mannose synthase (DPM1) mutations define congenital disorder of glycosylation Ie (CDG-Ie). J Clin Invest. 2000;105(2):191-198. https://doi.org/10.1172/JCI7302

20. Imbach T, Schenk B, Schollen E, Burda P, Stutz A, Grunewald S, Bailie NM, King MD, Jaeken 
483

484

485

486

487

488

489

490

491

492

493

494

495

496

J, Matthijs G, Berger EG, Aebi M, Hennet T. Deficiency of dolichol-phosphate-mannose synthase1 causes congenital disorder of glycosylation type Ie. J Clin Invest. 2000;105(2):233-239. https://doi.org/10.1172/JCI8691

21. Baksi K, Zhang Z, Banerjee A, Banerjee DK. Cloning and expression of mannosylphospho dolichol synthase from bovine adrenal medullary capillary endothelial cells. Glycoconj J. 2009;26(6):635-645. https://doi.org/10.1007/s10719-008-9214-9

22. Zhang Z, Banerjee A, Baksi K, Banerjee DK. Mannosylphospho dolichol synthase overexpression supports angiogenesis. Biocatal Biotransformation. 2010;28(1):90-98. https://doi.org/10.3109/10242420903411629

23. Banerjee DK. N-glycans in cell survival and death: cross-talk between glycosyltransferases. Biochim Biophys Acta. 2012;1820(9):1338-1346. https://doi.org/10.1016/j.bbagen.2012.01.013 24. Baksi K, Zhang Z, Banerjee A, Serrano JE, Perez LE, Linares L, Seijo A, Sanchez N, Katiyar U, Banerjee DK. Silencing Mannosylphospho Dolichol synthase with shRNA impacts differentiation of capillary endothelial cells. FASEB J. 2016;30:844. https://doi.org/10.1096/fasebj.30.1 supplement.844.1

25. Chandrashekar DS, Bashel B, Balasubramanya SA, Creighton CJ, Ponce-Rodriguez I, Chakravarthi BVSK, Varambally S. UALCAN: A portal for facilitating tumor subgroup gene expression and survival analyses. Neoplasia. 2017;19(8):649-658. https://doi.org/10.1016/j.neo.2017.05.002

26. Asplund A, Edqvist PH, Schwenk JM, Pontén F. Antibodies for profiling the human proteomeThe Human Protein Atlas as a resource for cancer research. Proteomics. 2012;12(13):2067-2077. https://doi.org/10.1002/pmic.201100504

27. Tang Z, Li C, Kang B, Gao G, Li C, Zhang Z. GEPIA: a web server for cancer and normal gene expression profiling and interactive analyses. Nucleic Acids Res. 2017;45(W1):W98-W102. https://doi.org/10.1093/nar/gkx247

28. Gao J, Aksoy BA, Dogrusoz U, Dresdner G, Gross B, Sumer SO, Sun Y, Jacobsen A, Sinha R, Larsson E, Cerami E, Sander C, Schultz N. Integrative analysis of complex cancer genomics and clinical profiles using the cBioPortal. Sci Signal. 2013;6(269):pl1. https://doi.org/10.1126/scisignal.2004088

29. Zhou Y, Zhou B, Pache L, Chang M, Khodabakhshi AH, Tanaseichuk O, Benner C, Chanda SK. Metascape provides a biologist-oriented resource for the analysis of systems-level datasets. Nat commun. 2019;10(1):1523. https://doi.org/10.1038/s41467-019-09234-6

30. Oliveira-Ferrer L, Legler K, Milde-Langosch K. Role of protein glycosylation in cancer metastasis. Semin Cancer Biol. 2017;44:141-152. https://doi.org/10.1016/j.semcancer.2017.03.002

31. Cheng WK, Oon CE. How glycosylation aids tumor angiogenesis: An updated review. Biomed Pharmacother. 2018;103:1246-1252. https://doi.org/10.1016/j.biopha.2018.04.119

32. Yap NY, Rajandram R, Ng KL, Pailoor J, Fadzli A, Gobe GC. Genetic and Chromosomal Aberrations and Their Clinical Significance in Renal Neoplasms. Biomed Res Int. 2015;2015:476508. https://doi.org/10.1155/2015/476508

33. Albertson DG. Gene amplification in cancer. Trends Genet. 2006;22:447-55. https://doi.org/ 
10.1016/j.tig.2006.06.007

34. Klein G, Klein E. Conditioned tumorigenicity of activated oncogenes. Cancer Res. 1986;46: 3211-3224.

35. Albertson DG, Collins C, McCormick F, Gray JW. Chromosome aberrations in solid tumors. Nat Genet. 2003;34:369-376. https://doi.org/10.1038/ng1215

36. Manos EJ, Kim ML, Kassis J, Chang PY, Wells A, Jones DA. Dolichol-phosphate-mannose3 (DPM3)/prostin-1 is a novel phospholipase C-gamma regu- lated gene negatively associated with prostate tumor invasion. Oncogene. 2001;20(22):2781-2790. https://doi.org/10.1038/sj.onc.1204379

37. Kousvelari EE, Banerjee DK, Murty L, Baum BJ. N-linked protein glycosylation in the rat parotid gland during aging. Mech Aging Dev. 1988;42(2):173-181. https://doi.org/ 10.1016/00476374(88)90072-3

38. Nozaki M, Ohishi K, Yamada N, Kinoshita T, Nagy A, Takeda J. Developmental abnormalities of glycophosphatidylinositol-anchor deficient embryos revealed by Crc/loxP system. Lab Invest. 1999;79(3):293-299.

39. Haeuptle MA, Hennet T. Congenital disorders of glycosylation: an update on defects affecting the biosynthesis of dolichol-linked oligosaccharides. Hum Mutat. 2009;30(12):1628-1641. https://doi.org/10.1002/humu.21126

40. Villanueva A. Hepatocellular carcinoma. N Engl J Med. 2019;380(15):1450-1462. https://doi.org/10.1056/NEJMra1713263

41. Ihara Y, Yoshimura M, Miyoshi E, Nishikawa A, Sultan AS, Toyosawa S, Ohnishi A, Suzuki M, Yamamura K, Ijuhin N, Taniguchi N. Ectopic expression of N-acetylglucosaminyltransferase III in transgenic hepatocytes disrupts apolipoprotein B secretion and induces aberrant cellular morphology with lipid storage. Proc Natl Acad Sci USA. 1998;95(5):2526-2530. https://doi.org/10.1073/pnas.95.5.2526

42. Lee J, Song EY, Chung TW, Kang SK, Kim KS, Chung TH, Yeom YI, Kim CH. Hyperexpression of $\mathrm{N}$-acetylglucosaminyltransferase-III in liver tissues of transgenic mice causes fatty body and obesity through severe accumulation of Apo A-I and Apo B. Arch Biochem Biophys. 2004;426(1):18-31. https://doi.org/ 10.1016/j.abb.2003.12.039

43. Ono M, Hakomori S. Glycosylation defining cancer cell motility and invasiveness. Glycoconj J. 2003;20(1):71-78. https://doi.org/10.1023/B:GLYC.0000018019.22070.7d

44. Burgess JB, Baenziger JU, Brown WR. Abnormal surface distribution of the human asialoglycoprotein receptor in cirrhosis. Hepatology. 1992;15(4):702-706. https://doi.org/ 10.1002/hep.1840150425

45. Sawamura T, Nakada H, Hazama H, Shiozaki Y, Sameshima Y, Tashiro Y. Hyperasialoglycoproteinemia in patients with chronic liver-diseases and or liver-cell carcinomaasialoglycoprotein receptor in cirrhosis and liver-cell carcinoma. Gastroenterology. 1984;87(6):1217-1221.

46. Clerc F, Reiding KR, Jansen BC, Kammeijer GSM, Bondt A, Wuhrer M. Human plasma proteinN-glycosylation. Glycoconj J. 2016;33(3):309-343. https://doi.org/10.1007/s10719-0159626-2 
47. Cancer Genome Atlas Research Network. Electronic address: wheeler@bcm.edu; Cancer Genome Atlas Research Network. Comprehensive and integrative genomic characterization of hepatocellular carcinoma. Cell. 2017;169(7):1327-1341.e23. https://doi.org/10.1016/j.cell.2017.05.046 48. Kuo HH, Lin RJ, Hung JT, Hsieh CB, Hung TH, Lo FY, Ho MY, Yeh CT, Huang YL, Yu J, $\mathrm{Yu}$ AL. High expression FUT1 and B3GALT5 is an independent predictor of post-operative recurrence and survival in hepatocellular carcinoma. Sci Rep. 2017;7(1):10750. https://doi.org/10.1038/s41598-017-11136-w

49. Sun J, Thurin J, Cooper HS, Wang P, Mackiewicz M, Steplewski Z, Blaszczyk-Thurin M. Elevated expression of H type GDP-L-fucose: beta-D-galactoside alpha-2-L-fucosyltransferase is associated with human colon adenocarcinoma progression. Proc Natl Acad Sci U S A. 1995;92(12):5724-5728. https://doi.org/ 10.1073/pnas.92.12.5724

50. Tateishi R, Yoshida H, Matsuyama Y, Mine N, Kondo Y, Omata M. Diagnostic accuracy of tumor markers for hepatocellular carcinoma: a systematic review. Hepatol Int. 2008;2(1):17-30. https://doi.org/ 10.1007/s12072-007-9038-x 51. Marrero JA, Feng Z, Wang Y, Nguyen MH, Befeler AS, Roberts LR, Reddy KR, Harnois D, Llovet JM, Normolle D, Dalhgren J, Chia D, Lok AS, Wagner PD, Srivastava S, Schwartz M. Alpha-fetoprotein, des-gamma carboxyprothrombin, and lectin-bound alpha-fetoprotein in early hepatocellular carcinoma. Gastroenterology. 2009;137(1):110-8. https://doi.org/ 10.1053/j.gastro.2009.04.005

52. Marrero JA, Su GL, Wei W, Emick D, Conjeevaram HS, Fontana RJ, Lok AS. Des-gamma carboxyprothrombin can differentiate hepatocellular carcinoma from non-malignant chronic liver disease in American patients. Hepatology. 2003;37:1114-21. https://doi.org/10.1053/jhep.2003.50195

53. Hippo Y, Watanabe K, Watanabe A, Midorikawa Y, Yamamoto S, Ihara S, Tokita S, Iwanari H, Ito Y, Nakano K, Nezu J, Tsunoda H, Yoshino T, Ohizumi I, Tsuchiya M, Ohnishi S, Makuuchi M, Hamakubo T, Kodama T, Aburatani H. Identification of soluble NH2-terminal fragment of glypican-3 as a serological marker for early-stage hepatocellular carcinoma. Cancer Res. 2004;64(7):2418-23. https://doi.org/10.1158/0008-5472.can-03-2191 
606

607

608

609

610

611

612

613

614

615

616

617

618

619

620

621

622

623

624

625

626

627

628

629

630

631

632

633

634

635

636

637

638

639

640

641

642

643

644

645

646

\section{Figure legend}

Figure 1. Transcriptional expressions of (A) DPM1, (B) DPM2 and (C) DPM3 in different types of cancer diseases (UALCAN database). Blue: Normal; Red: Tumor.

Abbreviations: BLCA, Bladder urothelial carcinoma; BRCA, Breast invasive carcinoma; CESC, Cervical squamous cell carcinoma; $\mathrm{CHOL}$, Cholangiocarcinoma; COAD, Colon adenocarcinoma; ESCA, Esophageal carcinoma; GBM, Glioblastoma multiforme; HNSC, Head and Neck squamous cell carcinoma; KICH, Kidney chromophobe; KIRC, Kidney renal clear cell carcinoma; KIRP, Kidney renal papillary cell carcinoma; LIHC, Liver hepatocellular carcinoma; LUAD, Lung adenocarcinoma; LUSC, Lung squamous cell carcinoma; PAAD, Pancreatic adenocarcinoma; PRAD, Prostate adenocarcinoma; PCPG, Pheochromocytoma and Paraganglioma; READ, Rectum adenocarcinoma; SARC, Sarcoma; SKCM, Skin cutaneous melanoma; THCA, Thyroid carcinoma; THYM, Thymoma; STAD, Stomach adenocarcinomna; UCEC, Uterine corpus endometrial carcinoma.

Figure 2. mRNA and protein expressions of DPMS in HCC and normal liver tissues. (A-C) mRNA expressions of DPM1, DPM2 and DPM3 in HCC tissues compared to normal samples (UALCAN database). ${ }^{* * *} p<0.001$. (D-F) Representative immunohistochemistry images of DPM1, DPM2 and DPM3 in HCC tissues and normal liver tissues (Human Protein Atlas).

Figure 3. Association of mRNA expressions of DPMS with tumor grades and patients' individual cancer stages in HCC patients (UALCAN). (A-C) Association of mRNA expressions of DPM1, DPM2 and DPM3 with tumor grades in HCC patients. (D-F) Relationship between mRNA 
647 expressions of DPM1, DPM2 and DPM3 and individual cancer stages of HCC patients. *p $<0.05$, $648 * * \mathrm{p}<0.01, * * * \mathrm{p}<0.001$.

649 Figure 4. The mRNA expressions of DPM1/2/3 in normal liver samples and CLD samples. (A-C)

650 The expressions of DPM1/2/3 in normal and HBV-related liver cirrhosis. (D-F) The expressions 651 of DPM1/2/3 in normal and HCV-related liver cirrhosis. (G-I) The expressions of DPM1/2/3 in 652 normal and non-alcoholic steatohepatitis.

653 Abbreviations: HBV-LC, HBV-related liver cirrhosis; HCV-LC, HCV-related liver cirrhosis, 654 NASH, non-alcoholic steatohepatitis.

655 Figure 5. The prognostic value of different expressed DPM1, DPM2 and DPM3 in HCC patients 656 (GEPIA). (A-C) Overall survival curves of DPM1, DPM2 and DPM3. (D-F) Disease free survival 657 curves of DPM1, DPM2 and DPM3.

658 Figure 6. The assessment of the diagnosis effect among DPM1/2/3 and existing markers in normal 659 and HCC using the ROC curve. (A-C) ROC curves and AUC values of DPM1, DPM2 and DPM3 660 respectively. (D-F) ROC curves and AUC values of AFP, GPC3 and TGF $\beta 1$ respectively.

661 Figure 7. Genetic alterations in DPMS and their association with OS and DFS in HCC patients 662 (cBioPortal). (A) Summary of alterations in DPMS. (B) OncoPrint visual summary of alteration 663 on a query of DPMS. (C) Kaplan-Meier plots comparing OS in cases with/without DPMS gene 664 alterations. (D) Kaplan-Meier plots comparing DFS in cases with/without DPMS gene alterations. 665 Figure 8. The enrichment analysis of DPMS and their similar genes in HCC (Metascape). (A) 666 Heatmap of Gene Ontology (GO) enriched terms colored by p-values. (B) Network of GO enriched 667 terms colored by p-value, where terms containing more genes tend to have a more significant p668 value. (C) Heatmap of Kyoto Encyclopedia of Genes and Genomes (KEGG) enriched terms 669 colored by p-values. (D) Network of KEGG enriched terms colored by p-value, where terms 670 containing more genes tend to have a more significant p-value. (E) Protein-protein interaction 671 (PPI) network and three most significant MCODE components form the PPI network. (F) 672 Independent functional enrichment analysis of three MCODE components.

673 Figure 9. The mRNA expression levels of (A) DPM1, (B) DPM2 and (C) DPM3 in normal liver 674 cells and hepatoma cell lines. ${ }^{*} \mathrm{p}<0.05, * * * \mathrm{p}<0.001$. 


\section{Figure 1}

Transcriptional expressions of (A) DPM1, (B) DPM2 and (C) DPM3 in different types of cancer diseases (UALCAN database).

Blue: Normal; Red: Tumor. Abbreviations: BLCA, Bladder urothelial carcinoma; BRCA, Breast invasive carcinoma; CESC, Cervical squamous cell carcinoma; CHOL, Cholangiocarcinoma; COAD, Colon adenocarcinoma; ESCA, Esophageal carcinoma; GBM, Glioblastoma multiforme; HNSC, Head and Neck squamous cell carcinoma; KICH, Kidney chromophobe; KIRC, Kidney renal clear cell carcinoma; KIRP, Kidney renal papillary cell carcinoma; LIHC, Liver hepatocellular carcinoma; LUAD, Lung adenocarcinoma; LUSC, Lung squamous cell carcinoma; PAAD, Pancreatic adenocarcinoma; PRAD, Prostate adenocarcinoma; PCPG, Pheochromocytoma and Paraganglioma; READ, Rectum adenocarcinoma; SARC, Sarcoma; SKCM, Skin cutaneous melanoma; THCA, Thyroid carcinoma; THYM, Thymoma; STAD, Stomach adenocarcinomna; UCEC, Uterine corpus endometrial carcinoma. 
A

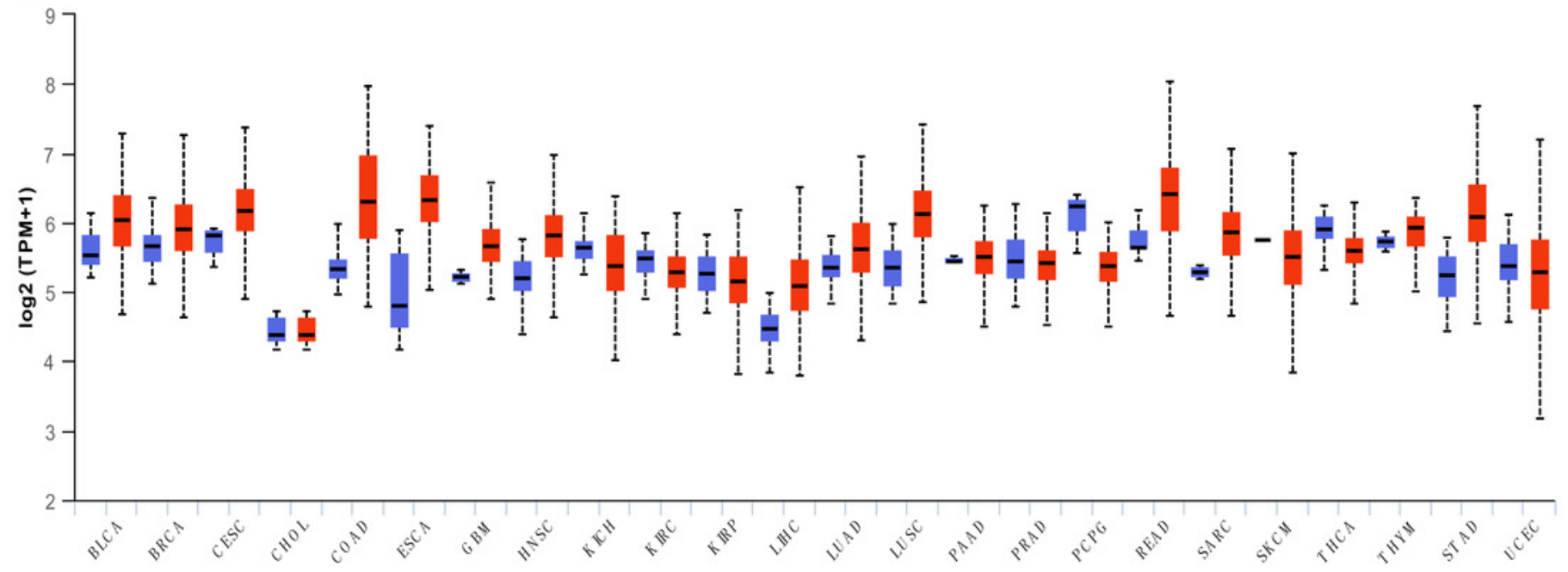

B
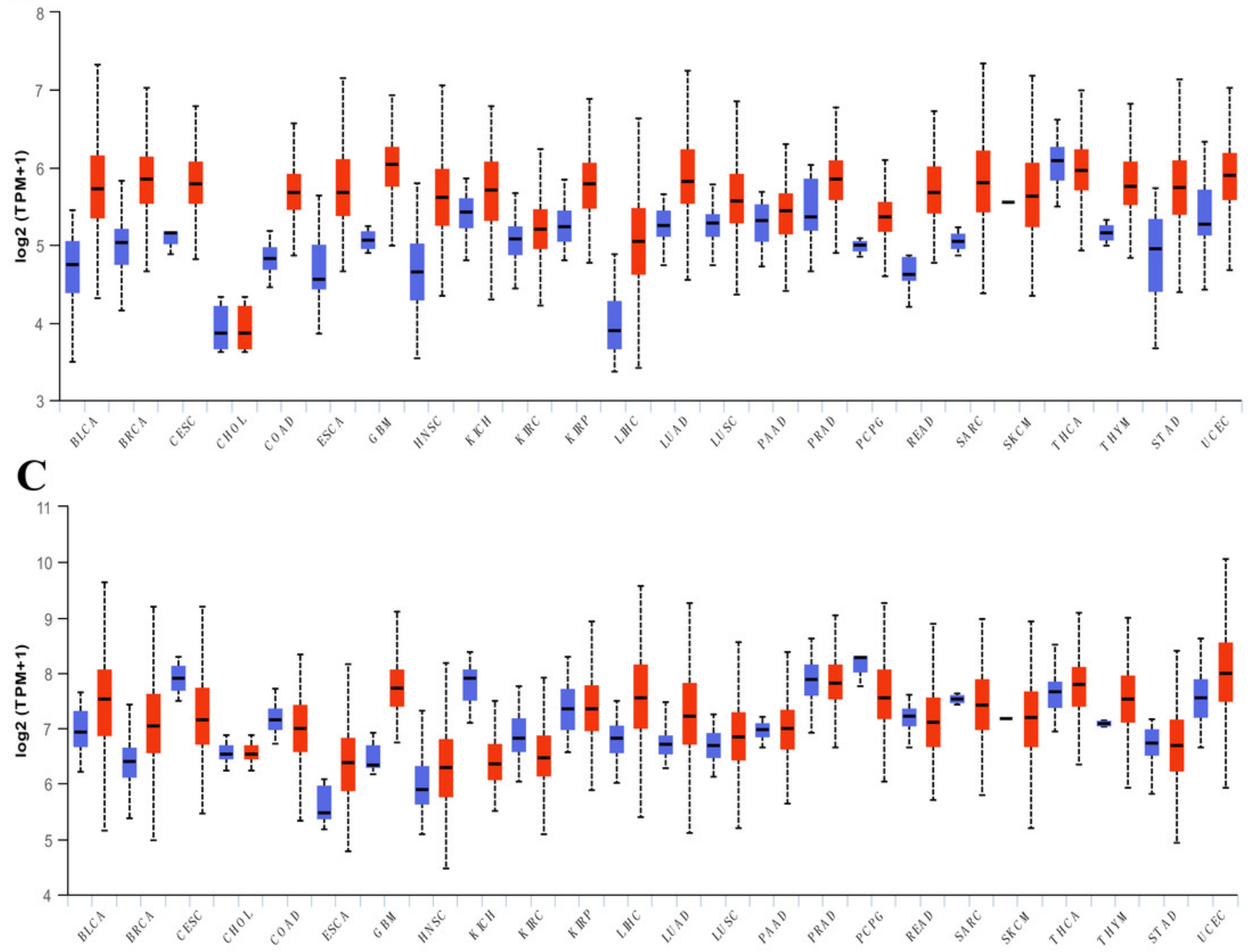
Figure 2

The mRNA and protein expressions of DPMS in HCC and normal liver tissues.

(A-C) mRNA expressions of DPM1, DPM2 and DPM3 in HCC tissues compared to normal samples (UALCAN database). ${ }^{* * *} p<0.001$. (D-F) Representative immunohistochemistry images of DPM1, DPM2 and DPM3 in HCC tissues and normal liver tissues (Human Protein Atlas).

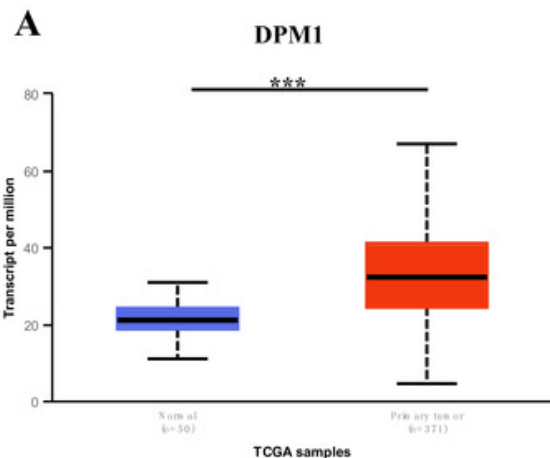

D

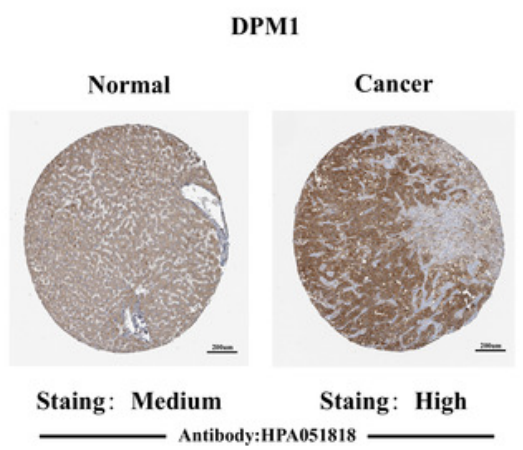

B

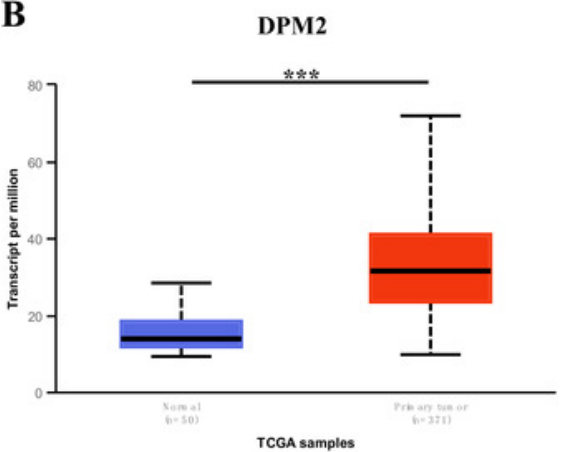

$\mathbf{E}$

DPM2

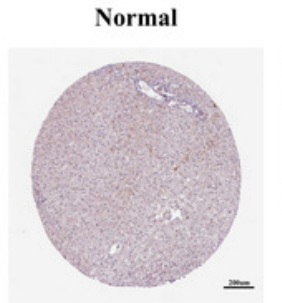

Staing: Not detected

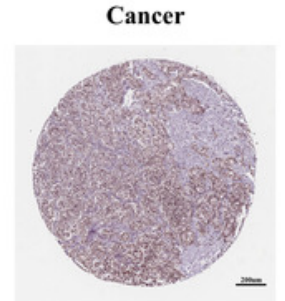

Staing: Medium

Antibody:HPA064023

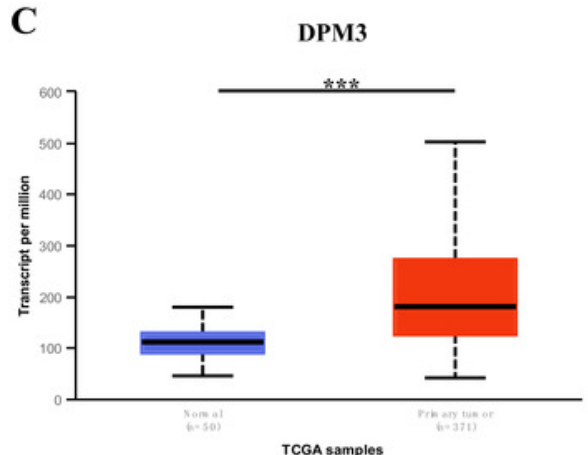

F

DPM3

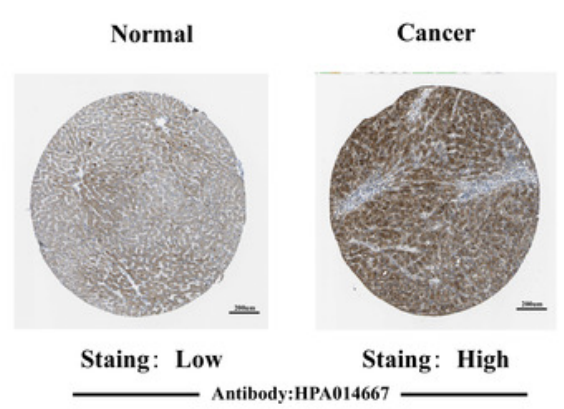


Figure 3

Association of mRNA expressions of DPMS with tumor grades and patients' individual cancer stages in HCC patients (UALCAN).

(A-C) Association of mRNA expressions of DPM1, DPM2 and DPM3 with tumor grades in HCC patients. (D-F) Relationship between mRNA expressions of DPM1, DPM2 and DPM3 and individual cancer stages of HCC patients. ${ }^{*} p<0.05,{ }^{* *} p<0.01,{ }^{* * *} p<0.001$.

A
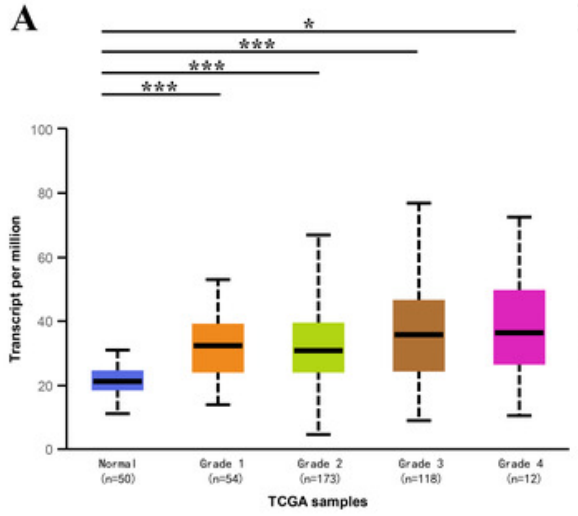

D
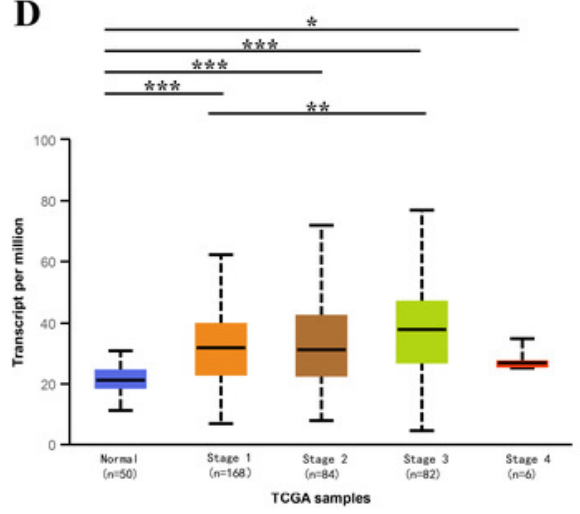

$\mathbf{E}$
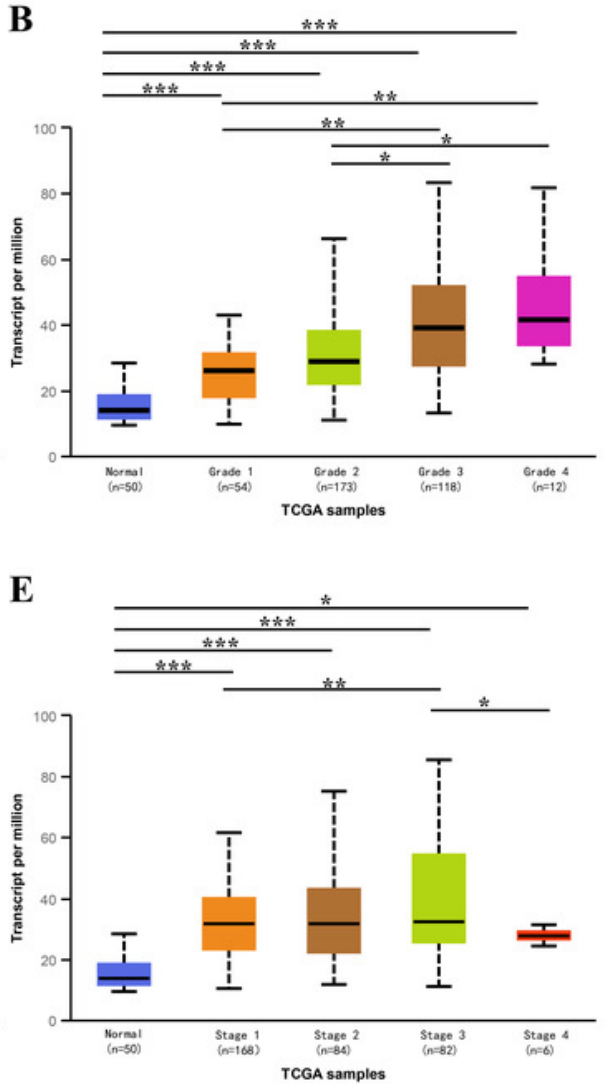

C
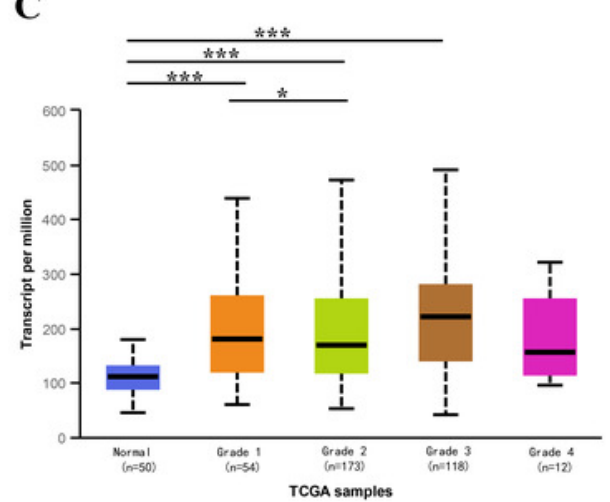

F
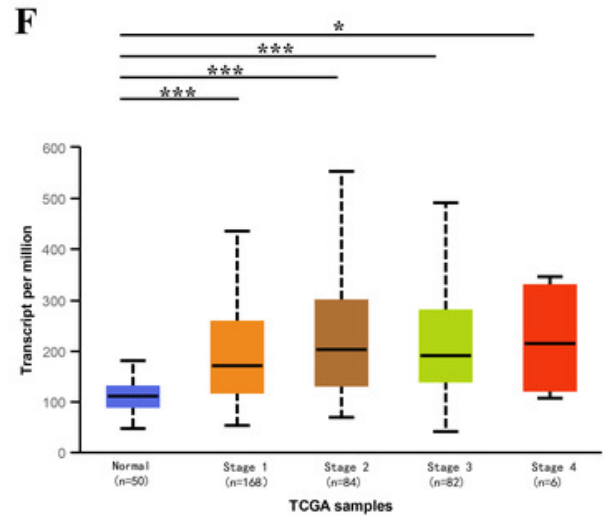
Figure 4

The mRNA expressions of DPM1/2/3 in normal liver samples and CLD samples.

(A-C) The expressions of DPM1/2/3 in normal and HBV-related liver cirrhosis. (D-F) The expressions of DPM1/2/3 in normal and HCV-related liver cirrhosis. (G-I) The expressions of DPM1/2/3 in normal and non-alcoholic steatohepatitis. Abbreviations: HBV-LC, HBV-related liver cirrhosis; HCV-LC, HCV-related liver cirrhosis; NASH, non-alcoholic steatohepatitis. 
A

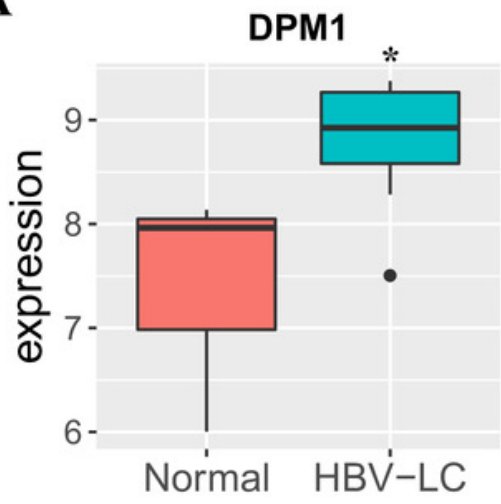

D

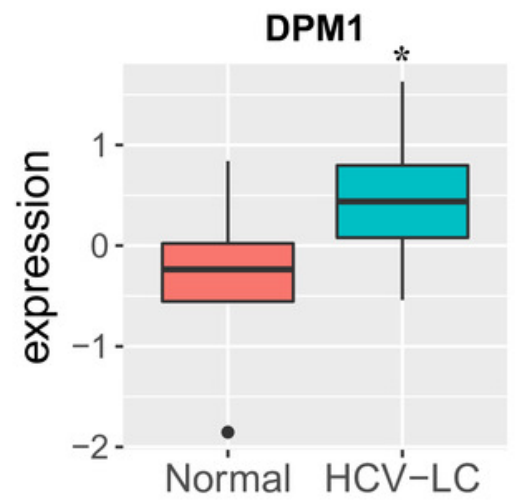

G

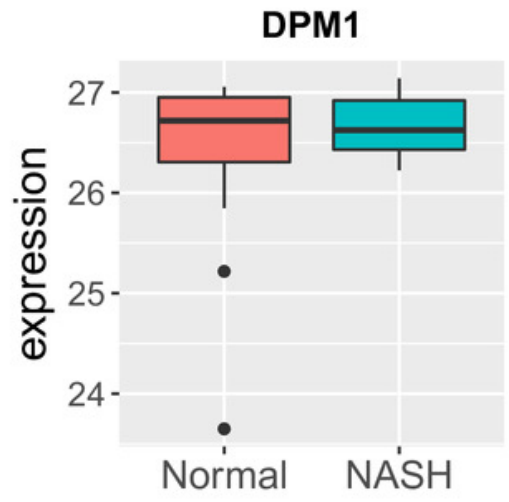

B

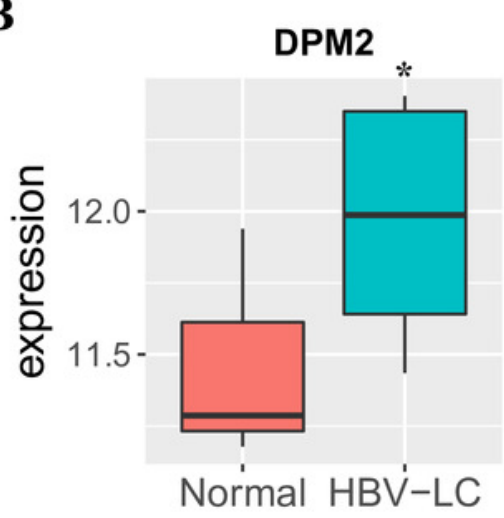

E

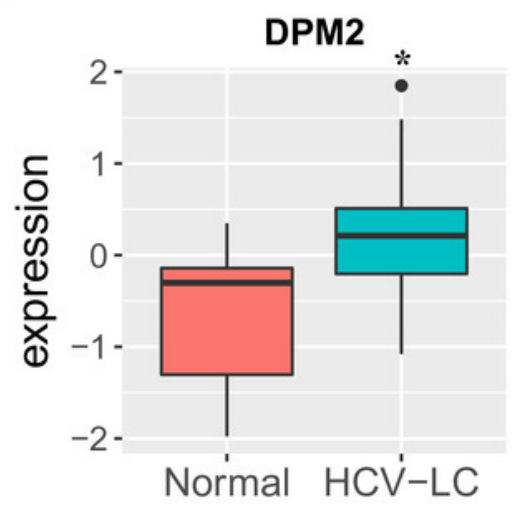

H

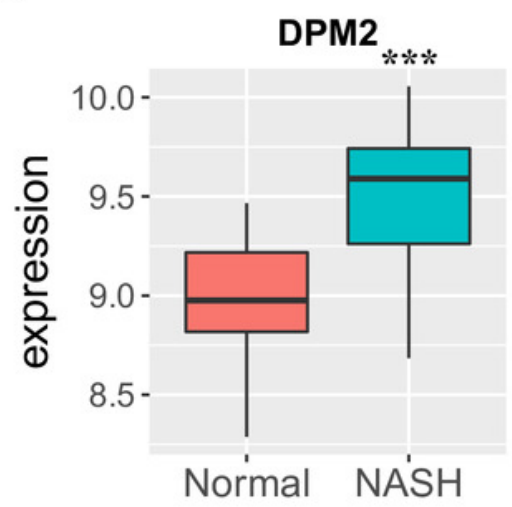

C

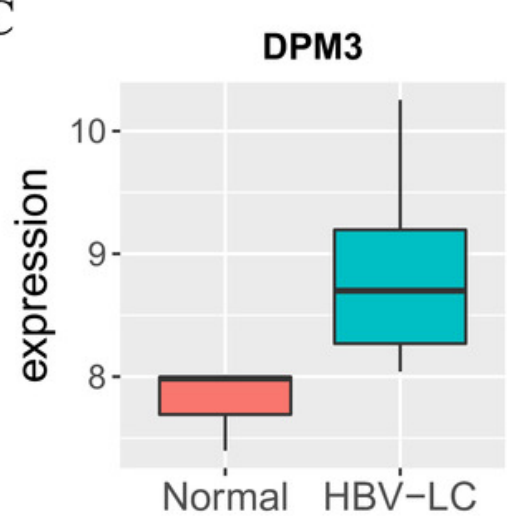

F

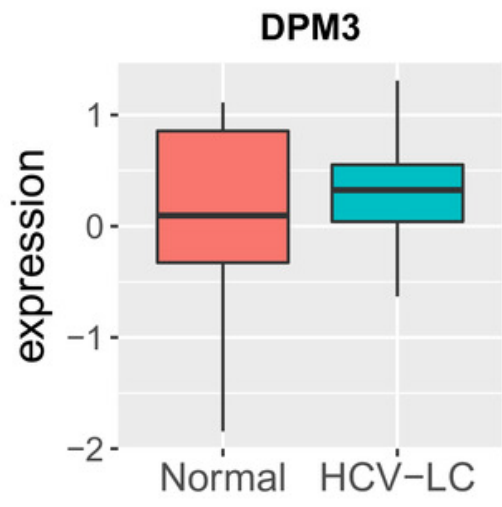

I

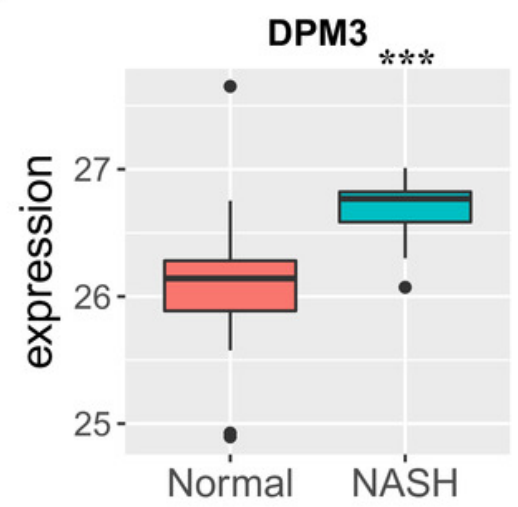


Figure 5

The prognostic value of different expressed DPM1, DPM2 and DPM3 in HCC patients (GEPIA).

(A-C) Overall survival curves of DPM1, DPM2 and DPM3. (D-F) Disease free survival curves of DPM1, DPM2 and DPM3.

A

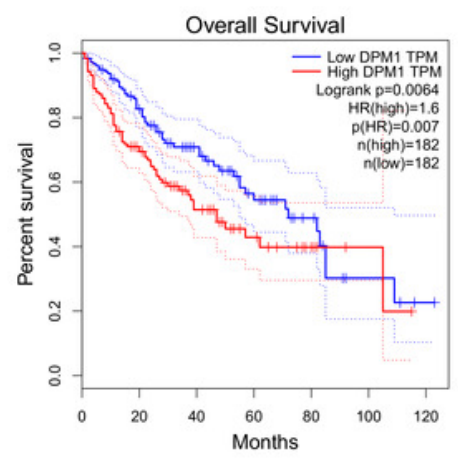

D

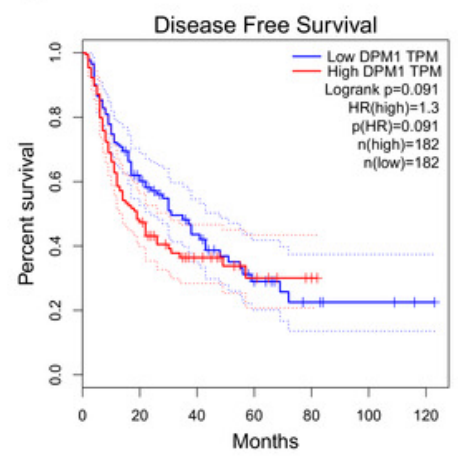

B

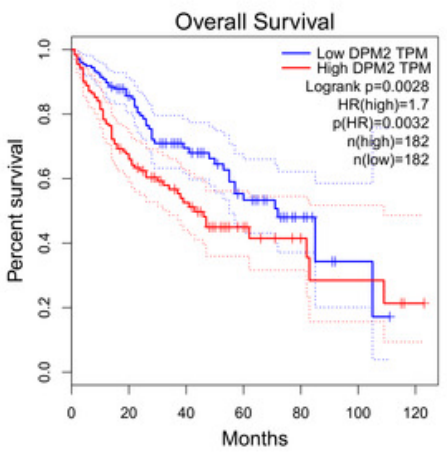

$\mathbf{E}$

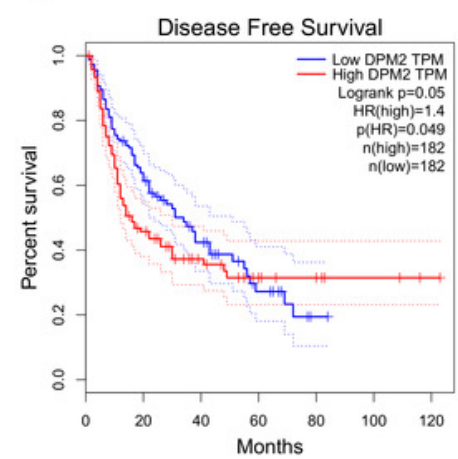

C

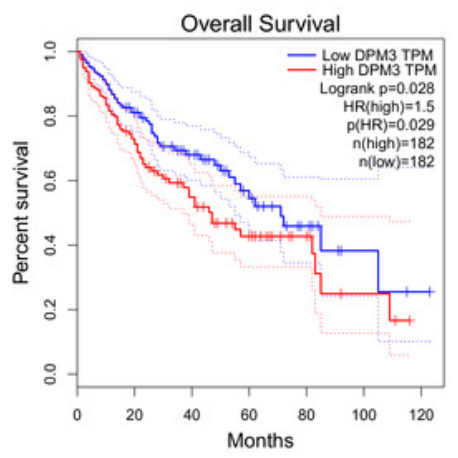

F

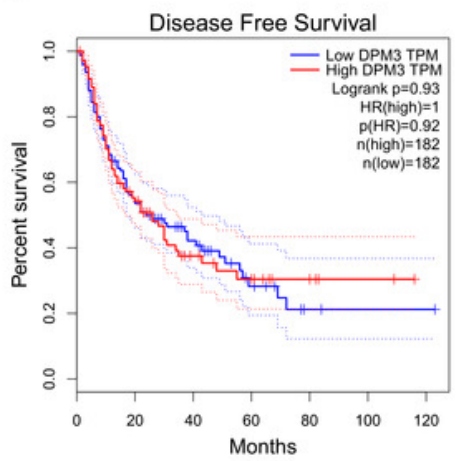




\section{Figure 6}

The assessment of the diagnosis effect among DPM1/2/3 and existing markers in normal and $\mathrm{HCC}$ using the ROC curve.

(A-C) ROC curves and AUC values of DPM1, DPM2 and DPM3 respectively. (D-F) ROC curves and AUC values of AFP, GPC3 and TGF $\beta 1$ respectively.

A

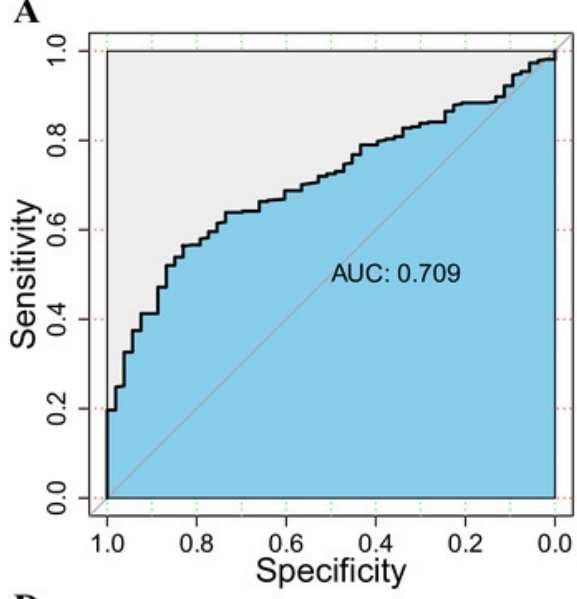

D

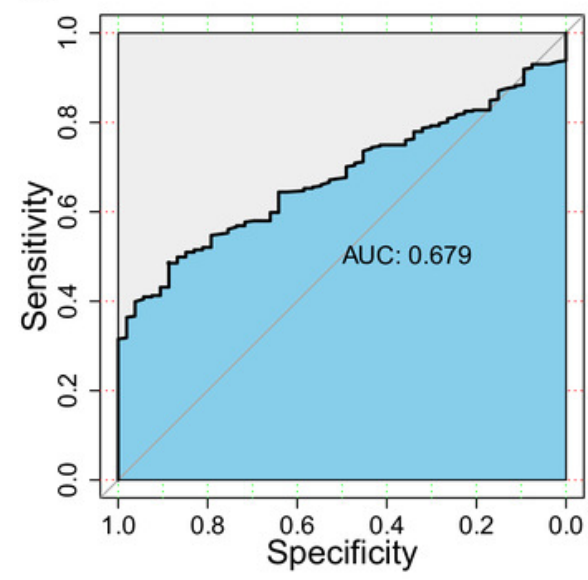

B

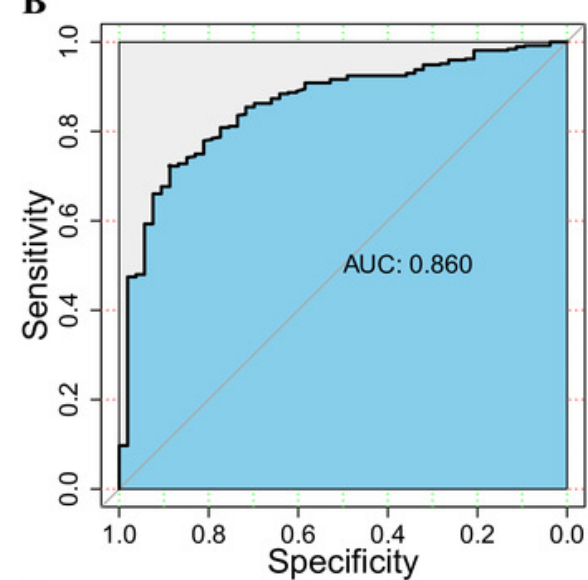

E

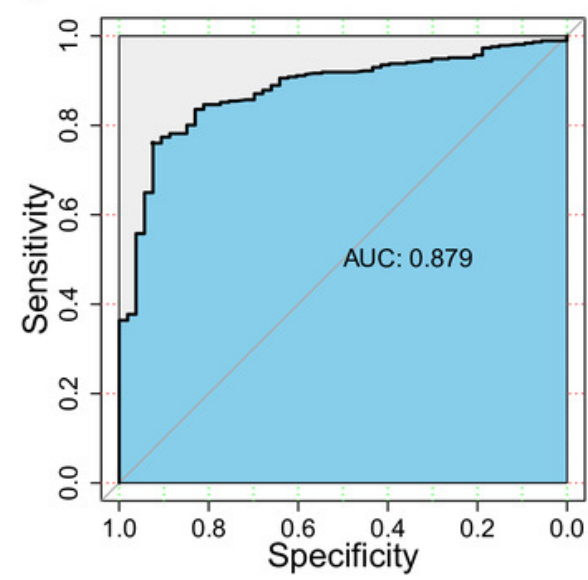

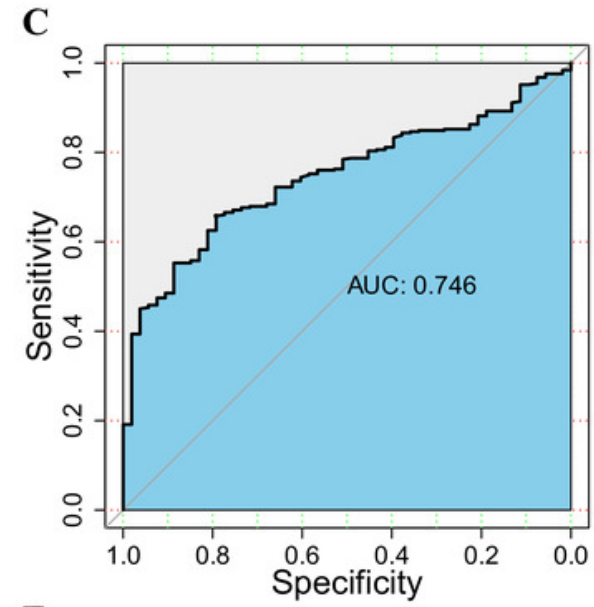

F

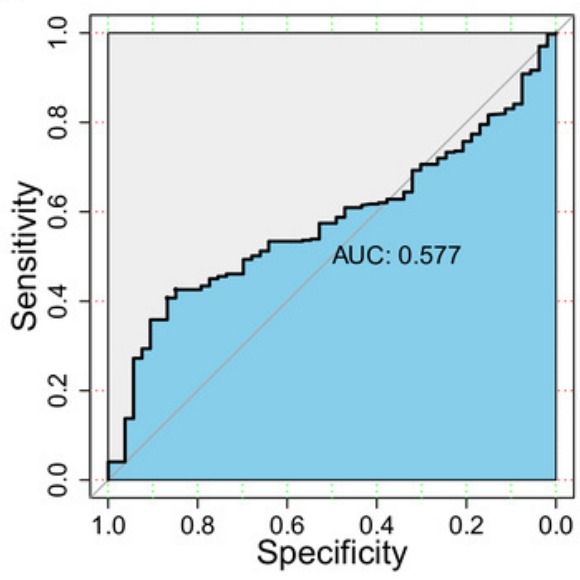




\section{Figure 7}

Genetic alterations in DPMS and their association with OS and DFS in HCC patients (cBioPortal).

(A) Summary of alterations in DPMS. (B) OncoPrint visual summary of alteration on a query of DPMS. (C) Kaplan-Meier plots comparing OS in cases with/without DPMS gene alterations. (D) Kaplan-Meier plots comparing DFS in cases with/without DPMS gene alterations.

A

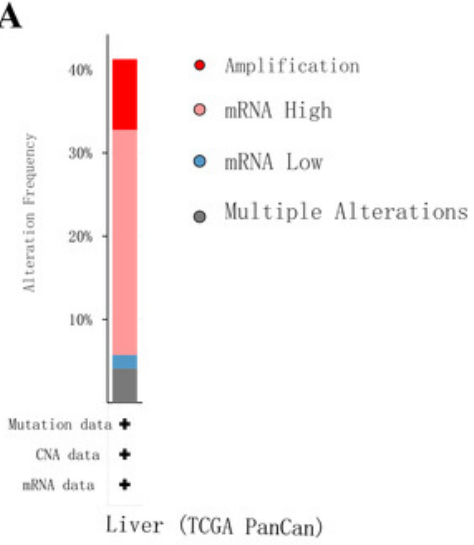

C

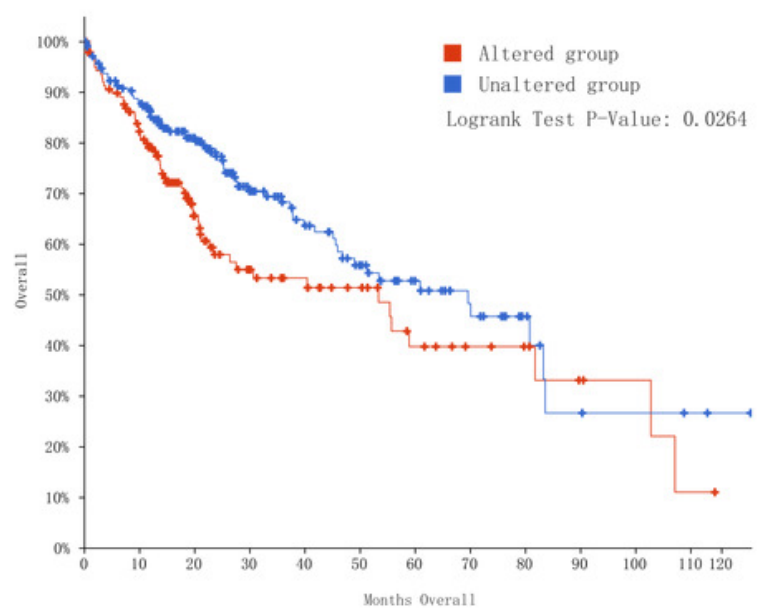

B

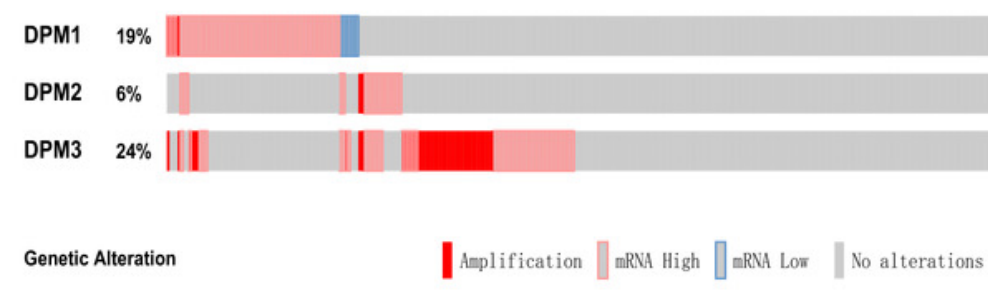

D

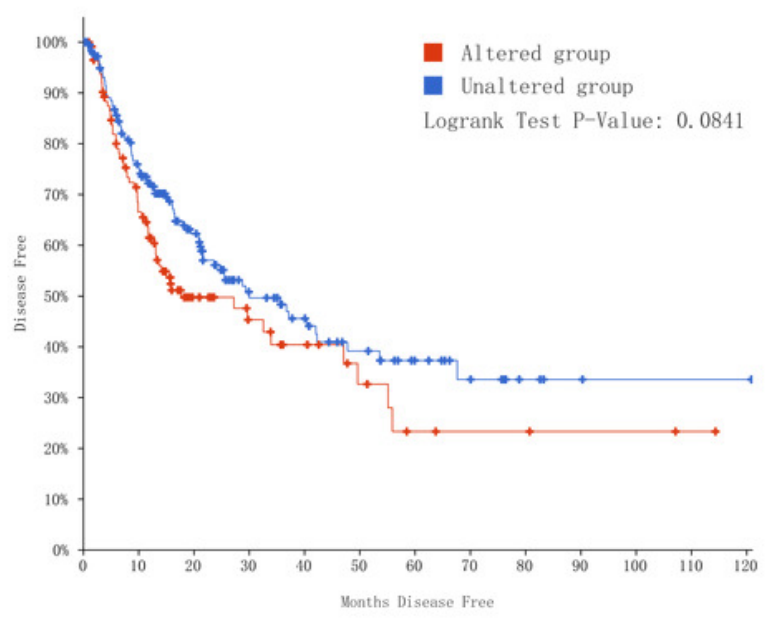




\section{Figure 8}

The enrichment analysis of DPMS and their similar genes in HCC (Metascape).

(A) Heatmap of Gene Ontology (GO) enriched terms colored by p-values. (B) Network of GO enriched terms colored by $p$-value, where terms containing more genes tend to have a more significant p-value. (C) Heatmap of Kyoto Encyclopedia of Genes and Genomes (KEGG) enriched terms colored by p-values. (D) Network of KEGG enriched terms colored by p-value, where terms containing more genes tend to have a more significant $p$-value. (E) Protein-protein interaction (PPI) network and three most significant MCODE components form the PPI network. (F) Independent functional enrichment analysis of three MCODE components. 
$\mathbf{A}$

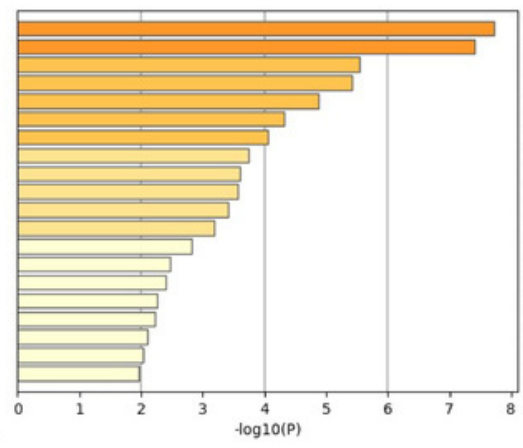

C

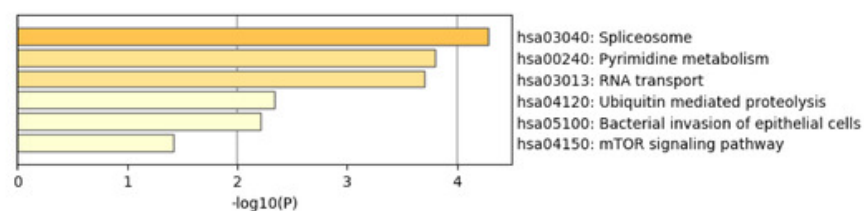

$\mathbf{E}$

GO:0070990: snRNP binding G:0034470: ncRNA processing GO:0006281: DNA repair O:0034708: methyltransferase complex O:0019080: viral gene expression GO:0008033: tRNA processing (1) .032006: regulation of TOR signaling (n) GO:0044452: nucleolar part

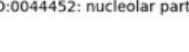

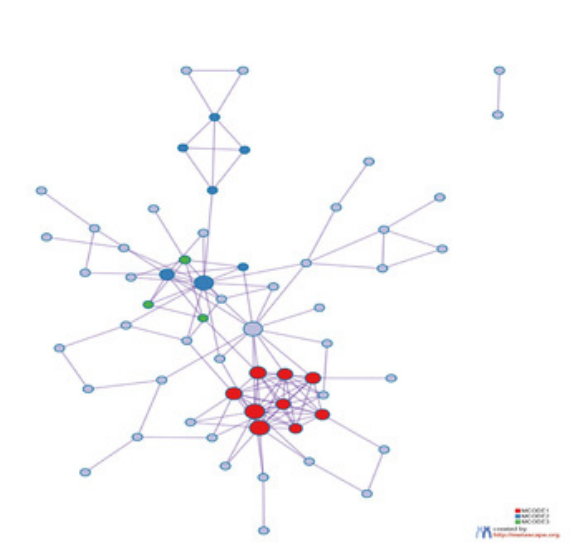

B express con 006169: non-recombinational repair :0071900: regulation of protein serine/threonine kinase activity . 0.0006353 : DNA-templated transcription, termination
D
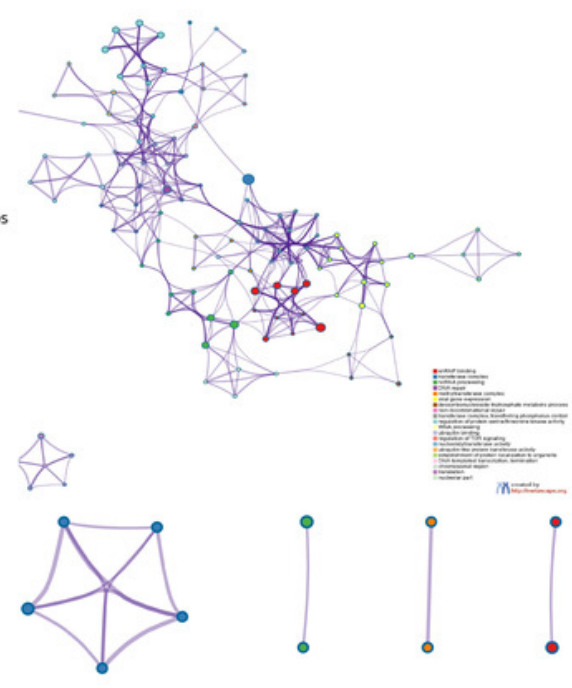

F

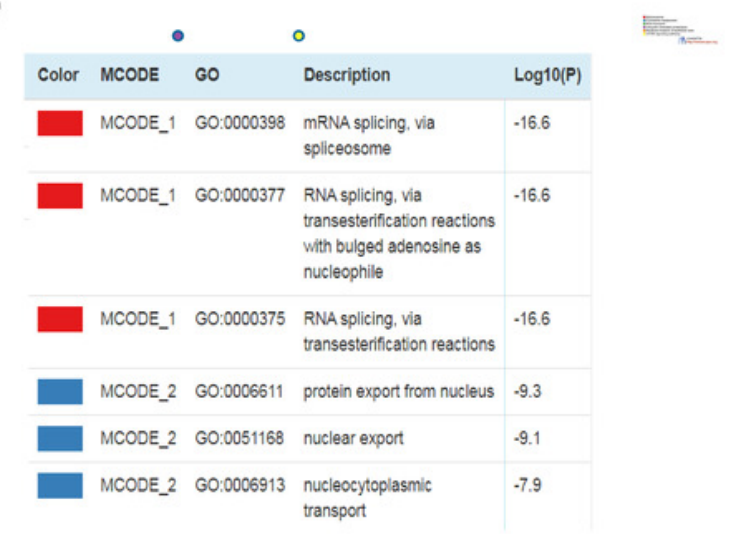


Figure 9

The mRNA expression levels of (A) DPM1, (B) DPM2 and (C) DPM3 in normal liver cells and hepatoma cell lines.

$* p<0.05, * * * p<0.001$.

$\mathbf{A}$

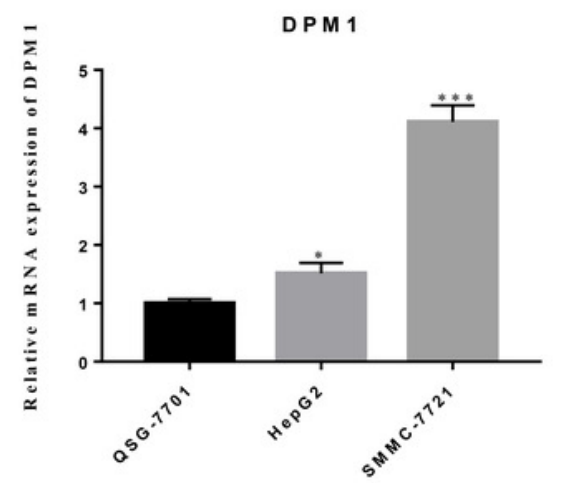

B

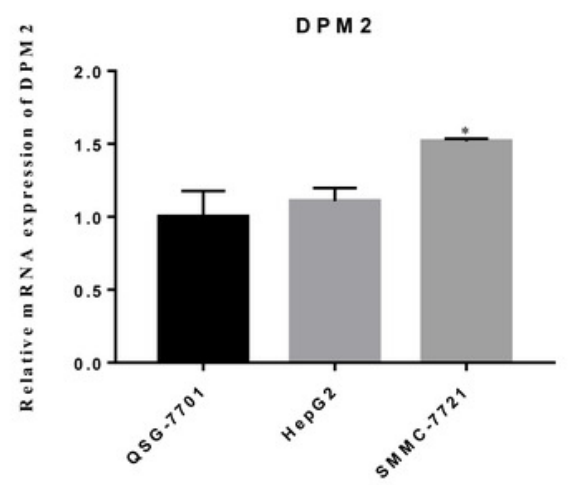

C

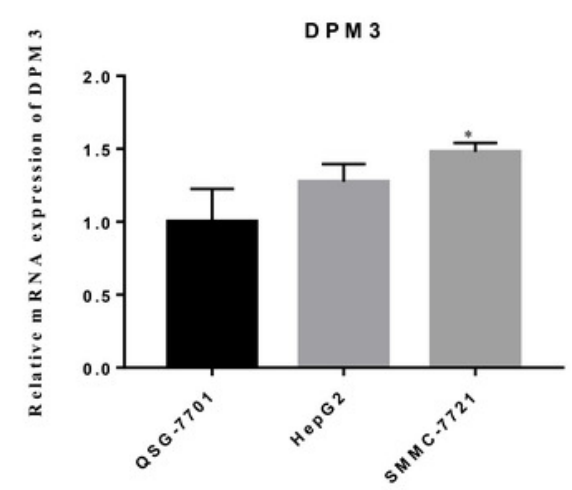


Table $\mathbf{1}$ (on next page)

Detailed information of the GEO datasets in this study. 
1

2 TABLE 1 Detailed information of the GEO datasets in this study.

\begin{tabular}{ccccccc}
\hline Datasets ID & Species & Data Type & Platform & Disease Type & $\begin{array}{c}\text { Normal } \\
\text { Number }\end{array}$ & $\begin{array}{c}\text { Patient } \\
\text { Number }\end{array}$ \\
\hline GSE114783 & $\begin{array}{c}\text { Homo } \\
\text { sapiens }\end{array}$ & $\begin{array}{c}\text { Expression } \\
\text { profiling } \\
\text { by array }\end{array}$ & GPL15491 & $\begin{array}{c}\text { HBV-related } \\
\text { liver cirrhosis }\end{array}$ & 3 & 10 \\
GSE128726 & Homo & $\begin{array}{c}\text { Expression } \\
\text { profiling } \\
\text { sapiens }\end{array}$ & GPL21185 & $\begin{array}{c}\text { HCV-related } \\
\text { liver cirrhosis }\end{array}$ & 9 & 10 \\
by array & & Expression \\
profiling & GPL14951 & $\begin{array}{c}\text { Non-alcoholic } \\
\text { steatohepatitis }\end{array}$ & 24 & 19 \\
\hline
\end{tabular}

3

4 
Table 2 (on next page)

Primers used for quantitative real-time PCR. 
1 TABLE 2 Primers used for quantitative real-time PCR.

\begin{tabular}{ccc}
\hline Gene & Primers & Sequences $(\mathbf{5} \rightarrow \mathbf{3})$ \\
\hline DPM1 & Forward & ACAGGAAGTTCAGATTATACCGAA \\
& Reverse & ATTCACCATAAACACGATCCACA \\
\hline DPM2 & Forward & GCATCCTTAGCCGCTACACT \\
& Reverse & GCGTTTGCCATGCCTAAGAG \\
\hline $\mathbf{D P M 3}$ & Forward & TCGCAGTGACCATGACGAAA \\
& Reverse & TTAGGCTGTCAGAAGCGCAG \\
& Forward & CGGCTACCACATCCAAGGAAG \\
\hline $\mathbf{1 8 S}$ & Reverse & AGCTGGAATTACCGCGGCT \\
\hline
\end{tabular}

2 


\section{Table 3 (on next page)}

The GO function enrichment analysis of DPM1/2/3 and their similar genes in HCC. 


\section{TABLE 3 The GO function enrichment analysis of DPM1/2/3 and their similar}

\section{2 genes in HCC.}

\begin{tabular}{|c|c|c|c|c|c|c|}
\hline GO & Category & Description & Count & $\%$ & $\log 10(P)$ & $\log 10(q)$ \\
\hline GO:0034470 & GO Biological Processes & ncRNA processing & 11 & 9.32 & -5.54 & -2.25 \\
\hline GO:0006281 & GO Biological Processes & DNA repair & 13 & 11.02 & -5.42 & -2.21 \\
\hline GO:0019080 & GO Biological Processes & viral gene expression & 7 & 5.93 & -4.32 & -1.25 \\
\hline GO:0009200 & GO Biological Processes & deoxyribonucleoside triphosphate metabolic process & 3 & 2.54 & -4.06 & -1.03 \\
\hline GO:0000726 & GO Biological Processes & non-recombinational repair & 5 & 4.24 & -3.74 & -0.8 \\
\hline GO:0071900 & GO Biological Processes & regulation of protein serine/threonine kinase activity & 10 & 8.47 & -3.57 & -0.79 \\
\hline GO:0008033 & GO Biological Processes & tRNA processing & 5 & 4.24 & -3.41 & -0.74 \\
\hline GO:0032006 & GO Biological Processes & regulation of TOR signaling & 4 & 3.39 & -2.81 & -0.38 \\
\hline GO:0072594 & GO Biological Processes & establishment of protein localization to organelle & 8 & 6.78 & -2.26 & -0.1 \\
\hline GO:0006353 & GO Biological Processes & DNA-templated transcription, termination & 3 & 2.54 & -2.23 & -0.09 \\
\hline GO:0006412 & GO Biological Processes & translation & 9 & 7.63 & -2.04 & 0 \\
\hline GO:1990234 & GO Cellular Components & transferase complex & 18 & 15.25 & -7.42 & -3.54 \\
\hline GO:0034708 & GO Cellular Components & methyltransferase complex & 6 & 5.08 & -4.89 & -1.74 \\
\hline GO:0061695 & GO Cellular Components & transferase complex, transferring phosphorus-containing groups & 7 & 5.93 & -3.6 & -0.79 \\
\hline GO:0098687 & GO Cellular Components & chromosomal region & 6 & 5.08 & -2.11 & -0.04 \\
\hline GO:0044452 & GO Cellular Components & nucleolar part & 4 & 3.39 & -1.96 & 0 \\
\hline GO:0070990 & GO Molecular Functions & snRNP binding & 4 & 3.39 & -7.73 & -3.54 \\
\hline GO:0043130 & GO Molecular Functions & ubiquitin binding & 4 & 3.39 & -3.19 & -0.63 \\
\hline GO:0016779 & GO Molecular Functions & nucleotidyltransferase activity & 4 & 3.39 & -2.47 & -0.25 \\
\hline GO:0019787 & GO Molecular Functions & ubiquitin-like protein transferase activity & 7 & 5.93 & -2.4 & -0.2 \\
\hline
\end{tabular}




\section{Table 4 (on next page)}

The KEGG function enrichment analysis of DPMS and their similar genes in HCC. 
1 TABLE 4 The KEGG function enrichment analysis of DPMS and their similar genes

2 in $\mathrm{HCC}$.

3

\begin{tabular}{|c|c|c|c|c|c|c|}
\hline GO & Category & Description & Count & $\%$ & $\log 10(P)$ & $\log 10(q)$ \\
\hline hsa03040 & $\begin{array}{l}\text { KEGG } \\
\text { Pathway }\end{array}$ & Spliceosome & 6 & 5.08 & -4.29 & -1.59 \\
\hline hsa00240 & $\begin{array}{l}\text { KEGG } \\
\text { Pathway }\end{array}$ & Pyrimidine metabolism & 5 & 4.24 & -3.8 & -1.57 \\
\hline hsa03013 & $\begin{array}{l}\text { KEGG } \\
\text { Pathway }\end{array}$ & RNA transport & 6 & 5.08 & -3.7 & -1.57 \\
\hline hsa04120 & $\begin{array}{l}\text { KEGG } \\
\text { Pathway }\end{array}$ & $\begin{array}{l}\text { Ubiquitin mediated } \\
\text { proteolysis }\end{array}$ & 4 & 3.39 & -2.34 & -0.55 \\
\hline hsa05100 & $\begin{array}{l}\text { KEGG } \\
\text { Pathway }\end{array}$ & $\begin{array}{l}\text { Bacterial invasion of } \\
\text { epithelial cells }\end{array}$ & 3 & 2.54 & -2.21 & -0.47 \\
\hline hsa04150 & $\begin{array}{l}\text { KEGG } \\
\text { Pathway }\end{array}$ & mTOR signaling pathway & 3 & 2.54 & -1.42 & 0 \\
\hline
\end{tabular}

4 